\title{
Mutant Cyclin F Impedes COPII Vesicle-Mediated ER-Golgi Trafficking and ER-Associated Degradation, Inducing ER Stress and Golgi Fragmentation in ALS/FTD
}

\section{Audrey Marie Genevieve RAGAGNIN ( $\nabla$ audrey.ragagnin@riken.jp )}

Macquarie University Faculty of Medicine and Health Sciences: Macquarie University Faculty of Medicine Health and Human Sciences https://orcid.org/0000-0002-2277-4029

\section{Vinod Sundaramoorthy}

Macquarie University Faculty of Medicine and Health Sciences: Macquarie University Faculty of Medicine Health and Human Sciences

\section{Marta Vidal}

Macquarie University Faculty of Medicine and Health Sciences: Macquarie University Faculty of Medicine Health and Human Sciences

\section{Cyril J Jagaraj}

Macquarie University Faculty of Medicine and Health Sciences: Macquarie University Faculty of Medicine Health and Human Sciences

\section{Sina Shadfar}

Macquarie University Faculty of Medicine and Health Sciences: Macquarie University Faculty of Medicine Health and Human Sciences

\section{Mariana Brocardo}

Macquarie University Faculty of Medicine and Health Sciences: Macquarie University Faculty of Medicine Health and Human Sciences

\section{Sonam Parakh}

Macquarie University Faculty of Medicine and Health Sciences: Macquarie University Faculty of Medicine Health and Human Sciences

\section{Anna Konopka}

Macquarie University Faculty of Medicine and Health Sciences: Macquarie University Faculty of Medicine Health and Human Sciences

\section{Natalie Grima}

Macquarie University Faculty of Medicine and Health Sciences: Macquarie University Faculty of Medicine Health and Human Sciences

\section{Shu Yang}

Macquarie University Faculty of Medicine and Health Sciences: Macquarie University Faculty of Medicine Health and Human Sciences 


\section{Stephanie Rayner}

Macquarie University Faculty of Medicine and Health Sciences: Macquarie University Faculty of Medicine Health and Human Sciences

\section{Kelly Williams}

Macquarie University Faculty of Medicine and Health Sciences: Macquarie University Faculty of Medicine Health and Human Sciences

\section{Lezanne Ooi}

University of Wollongong

\section{lan P Blair}

Macquarie University Faculty of Medicine and Health Sciences: Macquarie University Faculty of Medicine Health and Human Sciences

\section{Roger S Chung}

Macquarie University Faculty of Medicine and Health Sciences: Macquarie University Faculty of Medicine Health and Human Sciences

\section{Albert Lee}

Macquarie University Faculty of Medicine and Health Sciences: Macquarie University Faculty of Medicine Health and Human Sciences

\section{Julie Atkin}

Macquarie University Faculty of Medicine and Health Sciences: Macquarie University Faculty of Medicine Health and Human Sciences

\section{Research article}

Keywords: Cyclin F, ER-Golgi transport, ER stress, Amyotrophic lateral sclerosis, Frontotemporal Dementia

Posted Date: August 17th, 2021

DOI: https://doi.org/10.21203/rs.3.rs-783450/v1

License: (c) (1) This work is licensed under a Creative Commons Attribution 4.0 International License. Read Full License 


\section{Abstract}

\section{Background}

Mutations in the CCNF gene encoding cyclin F are associated with sporadic and familial amyotrophic lateral sclerosis (ALS) and frontotemporal dementia, but the underlying pathophysiological mechanisms are unknown. Proper functioning of the endoplasmic reticulum (ER) is essential for physiological cellular function.

\section{Methods}

We used human neuroblastoma SH-SY5Y and human embryonic kidney HEK293T cell lines and mouse primary neurons-overexpressing two familial ALS cyclin F mutants to examine whether mutant ALS/FTDassociated cyclin F perturbs key functions of the ER and Golgi compartments. Specific cellular assays were used to examine ER-Golgi transport (VSVG ${ }^{\text {t5045 }}$ ), the budding of vesicles from ER membranes and ER-associated degradation (ERAD). Immunocytochemistry was used to examine the morphology of the Golgi and ER-exit sites, and to detect ER stress and apoptosis. Western blotting was used to examine the content of vesicles budding from ER membranes and the interaction between Sec 31 and cyclin F. Flow cytometry was used to examine cell death.

\section{Results}

We demonstrated that mutant cyclin $\mathrm{F}$ inhibited protein transport from the ER to Golgi apparatus by a mechanism involving aberrant vesicle sorting from the ER. It also impeded ER-associated degradation, whereby misfolded ER proteins are ubiquitinated and degraded by the proteasome. This was associated with induction of ER stress and Golgi fragmentation, leading to apoptosis.

\section{Conclusion}

Together, these results demonstrate that ER dysfunction is a pathogenic pathway associated with ALS/FTD-variant cyclin F.

\section{Background}

Amyotrophic lateral sclerosis (ALS) is a fatal neurodegenerative disorder characterized by the progressive degeneration of both upper and lower motor neurons. ALS displays genetic and pathogenic overlap with the related condition frontotemporal dementia (FTD) in up to $15 \%$ of ALS patients [1, 2]. Approximately $10 \%$ of ALS cases are familial (FALS), which are clinically indistinguishable from sporadic ALS (SALS). Mutations in genes including SOD1, TARDBP, and FUS are present in FALS, and we recently identified missense mutations in the CCNF gene, encoding cyclin F, in patients with FALS/FTD [3]. Understanding early pathogenic mechanisms is important for designing effective therapeutic targets, but they remain virtually uncharacterised for cyclin F-associated ALS/FTD. 
CCNF[NM_001761.3] (also called FBXO1) encodes cyclin F [NP_001752.2], an orphan member of the cyclin protein family $[4,5]$. Whilst cyclins are important regulators of the cell cycle $[4,6]$, unlike most cyclins, cyclin F does not activate cyclin-dependent kinases (CDK). However, it is the founding member of a larger family of proteins containing a F-box domain [7]. This domain binds directly to S-phase kinaseassociated protein 1 (SKP1) which recruits cullin-1 (CUL1) to form the SCF (SKP1-CUL1-F-box protein) E3 ubiquitin-protein ligase complex, an integral component of the ubiquitin proteasome system (UPS) [7]. While other cyclins use the catalytic subunit of CDK to phosphorylate their substrates, cyclin F uses its Fbox to promote the ubiquitination of target substrates. Also, cyclin $\mathrm{F}$ contains two nuclear localization signals (NLS) at the C-terminus which facilitates its cellular localization in the nucleus [4]. Cyclin F contains a PEST sequence (enriched in proline, glutamic acid, serine and threonine) which controls its self-degradation through UPS-independent mechanisms [8]. Ubiquitination has important roles in protein transport by serving as a sorting signal for protein cargo and by controlling the activity of the trafficking machinery [9] and marking proteins for destruction [10]. We previously showed that ALS/FTD-linked CCNF p.S621G (c.1861A > G) and p.S195R (c.585T > G) mutations disrupt the UPS and impair ubiquitinmediated proteasomal degradation [3]. The p.S621G mutant cyclin F protein also disrupts Lys48-specific ubiquitination, leading to defects in the autophagic machinery $[11,12]$. Transient overexpression of mutant $\mathrm{S} 621 \mathrm{G}$ cyclin $\mathrm{F}$ in zebrafish larvae results in abnormal motor axon morphology, which correlates with motor dysfunction [13].

Defects in the proteostasis network are widely implicated in ALS/FTD $[14,15]$ and the endoplasmic reticulum (ER) and Golgi apparatus compartments play important roles in maintaining proteostasis. A major function of the ER is the production of secretory/transmembrane proteins and their subsequent transport to the Golgi, from where they are sorted to their final destinations. ER-Golgi transport includes multiple steps, and it is initiated when vesicles containing protein cargo bud from the ER coated in coat protein II (COPII) complex, consisting of Sec31, Sec13, Sec23, Sec24 and Sar1. After the budding of COPII vesicles at ER exit sites [16, 17], tethering, docking and fusion to target membranes at the Golgi follows $[18,19]$. The ER also plays an essential function in protein folding and quality control. ER-associated degradation (ERAD) ensures that only properly folded proteins are secreted, by retro-translocation of misfolded proteins to the cytosol for degradation by the UPS [20]. Ubiquitination of ERAD substrates ensures that they are recognised by the proteasome, but accumulating evidence suggests ubiquitination also performs a regulatory role in ERAD [21].

Dysfunction to the ER induces ER stress, the accumulation of misfolded/unfolded proteins within the ER, which triggers the unfolded protein response (UPR). Whilst initially protective, the UPR triggers apoptosis if prolonged or unresolved, hence ER stress is closely linked to cell survival. Similarly, proper functioning of the Golgi apparatus is closely linked to cellular viability and perturbations in this process are associated with apoptosis [22]. The unique morphology of the Golgi, with its tethered stacks of cisternae, is required for its function. However, the Golgi apparatus can undergo disassembly and fragmentation into condensed, tubulovesicular punctate structures when its functions are compromised or during pathological conditions $[23,24]$. 
In this study, we demonstrate that expression of mutant ALS/FTD cyclin F in neuronal cells perturbs key functions of the ER. Protein transport between the ER and Golgi apparatus was inhibited in mutant cyclin F expressing cells by a mechanism involving the formation of aberrant coat protein complex II (COPII) vesicles and/or ER-exit sites. This was accompanied by activation of ER stress, Golgi fragmentation, impairment of ERAD and induction of apoptosis. This study therefore reveals novel insights into the pathogenic mechanisms induced by cyclin F mutations in ALS/FTD, involving perturbations to both the ER and Golgi compartments.

\section{Materials \& Methods}

\section{Constructs}

Expression constructs encoding wild-type (WT) and mutant S621G or S195R CCNF CDNA fused to Cterminal mCherry, were generated by subcloning CCNF CDNA into pmCherry-C1 vector (Addgene: https://www.addgene.org/32975/) as previously described [3]. The point mutations in CCNF (S621G or S195R) were introduced using a Q5® Site-Directed Mutagenesis kit, according to the manufacturer's protocol (New England Biolabs E0554) [3]. Constructs encoding WT Venus or mutant single strand deglycosylation-dependant Venus (SS-ddVenus), where the C-terminus was fused to the unstable null Hong-Kong substrate (NHK-Venus; NHK-ddVenus) [25], were a kind gift from Dr Jess E Grotzke (Department of Immunobiology, Yale University School of Medicine, New Haven, USA) [26]. The mutant vesicular stomatitis viral glycoprotein (VSVG ${ }^{t 5045}$ ) fused to EGFP in the pEGFP-C1 vector was a kind gift from Dr Jennifer Lippincott-Schwartz (National Institutes of Health, Bethesda, USA).

\section{SH-SY5Y and HEK293T cell lines}

Undifferentiated human neuroblastoma SH-SY5Y and human embryonic kidney HEK293T cell lines (ATCC) were cultured for $24 \mathrm{~h}$ to $80 \%$ confluence at $37^{\circ} \mathrm{C}$ in a humidified atmosphere of $5 \% \mathrm{CO}_{2}$ in Dulbecco's modified Eagle's medium (DMEM; Gibco), supplemented with $10 \%(\mathrm{v} / \mathrm{v}$ ) heat-inactivated foetal bovine serum (Gibco). Authentication of cell line identities was confirmed via short tandem repeat profiling (Garvan Institute, Sydney). Cells were transfected for $48 \mathrm{~h}$ or $72 \mathrm{~h}$ with plasmids encoding pmCherry-C1 empty vector (EV), WT or mCherry-tagged mutant S621G or S195R CCNF, or co-transfected with plasmids encoding EV, mCherry-tagged WT or mutant CCNF with plasmids encoding GFP-tagged VSVG $^{\text {ts045 }}$, or Venus/ddVenus using Lipofectamine ${ }^{\text {Tw }} 2000$ (Invitrogen), following the manufacturer's instructions. Neuronal SH-SY5Y cells were used for immunocytochemistry studies, whereas HEK293T cells were used primarily for Western blotting studies because of their high transfection efficiency.

\section{Mouse primary cortical neurons}

Primary neurons were harvested from the cortex of C57BL/6 mouse embryos at embryonic day 16-18. The culture of primary neurons was performed as described previously [27]. Briefly, cortical tissue was dissected, cut into pieces under sterile conditions in Hanks' Balanced Salt solution (Gibco) and digested in 10 units $/ \mathrm{ml}$ papain (Sigma) in $0.2 \mathrm{mg} / \mathrm{ml}$ L-cysteine, $1 \mathrm{mM} \mathrm{CaCl}_{2}$ and $0.5 \mathrm{mM}$ EDTA (pH 8) in DMEM 
(Gibco) for $10 \mathrm{~min}$ at $37^{\circ} \mathrm{C}$. Cells were subsequently dissociated, resuspended in platting medium (Neurobasal medium [Gibco] supplemented with 10\% (v/v) heat-inactivated foetal bovine serum [Gibco], $2 \%(\mathrm{v} / \mathrm{v}) \mathrm{B}-27$ supplement [Gibco], 1\% (v/v) GlutaMAX ${ }^{\mathrm{TM}}$ [Gibco] and $100 \mu \mathrm{g} / \mathrm{ml}$ penicillin-streptomycin) and seeded for $12 \mathrm{~h}$ on $15 \mathrm{~mm}$ glass coverslips previously coated overnight with $0.1 \mathrm{mg} / \mathrm{ml}$ poly-D-lysine (Sigma). Cells were then incubated in neuronal medium (Neurobasal medium [Gibco] supplemented with $2 \%(\mathrm{v} / \mathrm{v})$ B-27 supplement [Gibco], $1 \%(\mathrm{v} / \mathrm{v})$ GlutaMAX ${ }^{\mathrm{TM}}$ [Gibco] and $100 \mu \mathrm{g} / \mathrm{ml}$ penicillin-streptomycin) at $37^{\circ} \mathrm{C}$ in a humidified atmosphere of $5 \% \mathrm{CO}_{2}$. Half of the medium was changed every three days. After 5 days in vitro, neurons were transfected with constructs encoding EV, WT or mutant S621G or S195R CCNF using Lipofectamine ${ }^{\mathrm{TM}} 2000$ (Invitrogen) following the manufacturer's instructions. Primary neurons were then incubated for $48 \mathrm{~h}$ before fixation in $4 \%$ paraformaldehyde (PFA) in $0.1 \mathrm{M}$ PBS.

\section{Immunocytochemistry}

SH-SY5Y and HEK293T cells grown on $13 \mathrm{~mm}$ coverslips were washed in $0.1 \mathrm{M}$ PBS (pH 7.2) and fixed in 4\% PFA in PBS for 10 min. After 3 washes in PBS, cells were permeabilised in $0.1 \%(\mathrm{v} / \mathrm{v})$ Triton X-100 in PBS for $5 \mathrm{~min}$ and the non-specific background staining was blocked, using $3 \%(\mathrm{w} / \mathrm{v})$ bovine serum albumin (BSA) in PBS for 45 min at room temperature with gentle rocking. Cells were then incubated overnight at $4^{\circ} \mathrm{C}$ with primary antibodies diluted in $1 \%(\mathrm{w} / \mathrm{v}) \mathrm{BSA}$ in PBS: polyclonal rabbit anti-calnexin (1:100; Abcam 22595) or monoclonal mouse anti-CHOP (1:50; Santa Cruz sc7351), anti-XBP1 M-186 (1:25; Santa Cruz sc-7160), anti-FLAG (1:500; Sigma F3165), anti-Sec31A (1:100; BD Biosciences 612351), or anti-GM130 (1:50; BD Transduction 610823). After rinsing, cells were incubated for $1 \mathrm{~h}$ at room temperature with gentle rocking and the appropriate secondary antibodies (diluted 1:250 in PBS) coupled to either Alexa 647 (Life Technology), Alexa 594 (Molecular Probes) or Alexa 488 (Life Technology). Cells were then washed as above and treated with $0.5 \mu \mathrm{g} / \mathrm{ml}$ Hoechst 33342 reagent (Sigma). After 3 washes in PBS, coverslips were mounted onto slides in fluorescent mounting medium (Dako) and cells were photographed with $20 \mathrm{x} / \mathrm{na}=0.8,40 \mathrm{x} / \mathrm{na}=1.3,63 \mathrm{x} / \mathrm{na}=1.4$ or $100 \mathrm{x} / \mathrm{na}=1.46$ objectives on a Zeiss LSM 880 inverted confocal laser-scanning microscope, equipped with a LSM-TPMT camera (Zeiss). Additional low-resolution images were acquired with $20 \mathrm{x} / \mathrm{na}=0.5,40 \mathrm{x} / \mathrm{na}=0.75,63 \mathrm{x} / \mathrm{na}$ $=1.4$ or $100 \mathrm{x} / \mathrm{na}=1.46$ objectives on an Axiolmager Z2 fluorescent microscope (Zeiss) equipped with a monochrome AxioCamHRm digital CCD camera (Zeiss).

\section{VSVG ${ }^{\text {ts045 }}$ transport assay}

SH-SY5Y cells co-transfected with constructs encoding cyclin F or VSVG ${ }^{\text {ts } 045}$ were incubated overnight at $40^{\circ} \mathrm{C}$ to accumulate VSVG ${ }^{\text {ts0 }} 45$ in the ER. Cycloheximide (Sigma) diluted $20 \mu \mathrm{g} / \mathrm{ml}$ in DMEM was then added and the cells were incubated at $32^{\circ} \mathrm{C}$ for $30 \mathrm{~min}$ to allow VSVG ${ }^{\text {ts } 045}$ to traffic to the Golgi. After one wash in PBS, samples were fixed in 4\% PFA in 0.1M PBS ( $\mathrm{pH} 7.2$ ) for $10 \mathrm{~min}$ and processed for immunocytochemistry as described above. At least 20 cells expressing both cyclin $F$ and VSVG ${ }^{\text {ts } 045}$ were photographed in each group. Mander's coefficient was calculated for each cell to determine the degree of colocalisation (where 0 indicated no colocalisation and 1 indicated total colocalisation) of VSVG ${ }^{\text {ts } 045}$ or 
cyclin F with either calnexin or GM130, using the JaCoP plugin [28] in ImageJ (http://rsbweb.nih.gov/ij/index.html). All experiments were performed in triplicate.

\section{Immunoprecipitation}

HEK293T cells transfected for 48h with constructs encoding cyclin F were removed by scraping in $400 \mu$ of non-denaturing lysis buffer (20 mM Tris-HCl, 150 mM NaCl, 2 mM EDTA, 1\% (v/v) NP40 pH 7.4, 1\% protease and $1 \%$ phosphatase inhibitor cocktail [Roche]) for $30 \mathrm{~min}$, followed by sonication for $10 \mathrm{~s}$ on ice and centrifugation at $14,000 \mathrm{~g}$ for $10 \mathrm{~min}$ at $4^{\circ} \mathrm{C}$. The concentration of protein in each lysate was calculated using a Pierce BCA Protein Assay Kit (Thermo Fisher Scientific) following the manufacturer's instructions. Cell lysate (500 $\mu \mathrm{g}$ of proteins) was incubated with either $2 \mu \mathrm{g}$ of rabbit polyclonal anti-FLAG antibody (Sigma F7425) or $2 \mu \mathrm{g}$ of mouse monoclonal anti-ubiquitin antibody (SantaCruz sc-8017) for $1 \mathrm{~h}$ at $4^{\circ} \mathrm{C}$ in a rotary shaker. Protein G/A Dynabeads (Thermo Fisher Scientific 10003D) were washed 3 times in lysis buffer and then incubated with each sample for $2 \mathrm{~h}$ at $4^{\circ} \mathrm{C}$ in a rotary shaker. The beads along with protein complexes were separated by placing the tubes in a magnetic rack. After rinsing in lysis buffer, the beads were mixed with $30 \mu$ of Laemmli buffer (BioRad 161-0747) and boiled at $95^{\circ} \mathrm{C}$ for 5 min before Western blotting was performed. Supernatants (10 $\mu \mathrm{g}$ of both input and flow-through) were mixed with Laemmli Buffer and NuPage sample reducing agent (Novex NP0009) and boiled at $95^{\circ} \mathrm{C}$ for $5 \mathrm{~min}$.

\section{In vitro budding assay}

A modified in vitro assay was used to analyse ER vesicle budding [23, 29]. Briefly, HEK293T cells cotransfected with constructs encoding cyclin F and VSVG ${ }^{\text {ts } 045}$ were incubated overnight at $40^{\circ} \mathrm{C}$ to accumulate VSVG ${ }^{\text {t5045 }}$ in the ER. Cells were then washed in PBS, resuspended in 5/90 buffer $(50 \mathrm{mM}$ HEPES and $90 \mathrm{mM}$ potassium acetate in demineralized water) and incubated with rat liver cytosol (Thermofisher) and an energy regenerating system ( $50 \mathrm{mM}$ creatine phosphate, $0.2 \mathrm{mg} / \mathrm{ml}$ creatine phosphokinase and $1 \mathrm{mM} \mathrm{ATP}$ ) at $32^{\circ} \mathrm{C}$ for $30 \mathrm{~min}$. Identical samples were incubated at $4^{\circ} \mathrm{C}$ to monitor non-specific ER fragmentation. The cells were removed by low-speed centrifugation at $4,000 \mathrm{~g}$ for $1 \mathrm{~min}$ at $4^{\circ} \mathrm{C}$, followed by $15,000 \mathrm{~g}$ for $1 \mathrm{~min}$, and budded vesicles were recovered by centrifugation at $100,000 \mathrm{~g}$ for $1 \mathrm{~h}$ at $4^{\circ} \mathrm{C}$ from the resulting supernatant. The levels of VSVG ${ }^{\mathrm{ts} 045}$ cargo in the budded vesicle fractions were quantified by Western blotting using anti-VSVG (1:1000; Sigma V4888) and anti-COPII (Sec23; 1:500; Pierce, Rockford, IL, PA1-069A) antibodies. The relative intensity of VSVG and COPII/Sec23 to $\beta$-actin was normalised to untreated cells.

\section{Western blotting}

HEK293T cells transfected with constructs encoding cyclin F for 48h were washed in PBS, lysed in 50 $\mathrm{mM}$ Tris- $\mathrm{HCl}(\mathrm{pH} 7.4), 150 \mathrm{mM} \mathrm{NaCl}, 0.1 \%$ (w/v) SDS, 1\% protease inhibitor cocktail (Sigma) and 1\% phosphatase inhibitor cocktail (Sigma). The supernatant was then cleared by centrifugation at $12,000 \mathrm{~g}$ for 10 min at $4^{\circ} \mathrm{C}$. The total amount of protein in each sample was quantified using a Pierce BCA Protein Assay Kit (Thermo Fisher Sci.), following the manufacturer's instructions. Proteins in the medium fraction were concentrated using Amicon Ultra $(0.5 \mathrm{ml})$ columns (Merck). Proteins $(10-20 \mu \mathrm{g})$ were separated on 
$7.5 \%, 4-15 \%$ or $4-20 \%$ gels (BioRad) and either stained with a Silver Stain kit (Thermo Fisher Sci.) or transferred onto nitrocellulose membranes according to the manufacturer's instructions (BioRad). Blots were pre-incubated in blocking solution containing $5 \%(\mathrm{w} / \mathrm{v})$ skim milk in Tris-buffered saline, followed by incubation overnight at $4^{\circ} \mathrm{C}$ in primary antibodies diluted in blocking solution: polyclonal rabbit anti-cyclin F (1:500; Santa Cruz sc952), anti-ubiquitin (1:1000; Abcam ab7780), anti-VSVG (1:1000; Sigma V4888), anti-COPII/Sec23 (1:500; Pierce, Rockford, IL, PA1-069A), or monoclonal mouse anti-Sec31 A (1:500; BD Biosciences 612351) or anti- $\beta$-actin (1:4000; Sigma A5441 [AC-15]) antibodies. After rinsing, blots were incubated in peroxidase-conjugated secondary antibodies (1:2000; Millipore) for one hour at room temperature or processed using fluorescent antibodies (Li-Cor Biosciences) following the manufacturer's instructions. Immunoreactivity was revealed using the Clarity ${ }^{T M}$ ECL Western Blotting Substrate (BioRad) and images were obtained either with the BioRad ChemiDoc MP system using Image Lab ${ }^{\text {TM }}$ software (BioRad) or with Odyssey ${ }^{\circledR}$ CLx and Image Studio software (Li-Cor Biosciences). The intensity of each band relative to $\beta$-actin was quantified using ImageJ.

\section{ERAD assay}

SH-SY5Y cells co-transfected with constructs encoding cyclin F and either NHK-Venus, NHK-ddVenus or SS-ddVenus for 48h, were fixed in 4\% PFA in 0.1M PBS, pH 7.2, and mounted as above. Images were acquired using an Axio Imager Z2 fluorescent microscope at 20x/na $=0.8$ magnification. At least 100 cells expressing cyclin $\mathrm{F}$ and Venus/ddVenus were scored as the percentage of NHK-Venus, NHKddVenus or SS-Venus fluorescent cells from three different experiments.

\section{Quantitative analysis of cells}

The percentage of cells expressing cyclin $\mathrm{F}$ with Golgi fragmentation was quantified from 10-30 primary neurons per group and from at least 50 SH-SY $5 Y$ or 100 HEK293T cells per group from $n=3$ independent experiments. Only cells where the Golgi structure was clearly visible were analysed. The Golgi was considered fragmented when at least 5 fragments were clearly visible. The area covered by the Golgi fragments was calculated using ImageJ.

The percentage of cells displaying nuclear immunoreactivity to CHOP or XBP1 was quantified from $30+$ primary neurons per group and at least $100+$ HEK293T cells per group expressing cyclin $F$ from $n \geq 3$ independent experiments. All analyses were performed blind.

\section{Quantitative analysis of apoptotic nuclei}

Apoptotic nuclei were defined as condensed when they were under $5 \mu \mathrm{m}$ in diameter or fragmented (multiple condensed Hoechst-positive structures in one cell) [30]. The percentage of apoptotic cells was quantified from 10-30 primary neurons per group or from at least 100 HEK293T cells per group expressing cyclin $F$ from $n \geq 3$ independent experiments. Cells undergoing cell division were excluded from analysis.

\section{Sytox Blue staining}


HEK293T transfected with cyclin F constructs for $72 \mathrm{~h}$ were harvested by adding trypsin for $1 \mathrm{~min}$ at room temperature. The cells were then collected in PBS, centrifuged at 1,200 rpm for 5 min and resuspended in $200 \mu$ of buffer containing $10 \mathrm{mM} \mathrm{HEPES}, 140 \mathrm{mM} \mathrm{NaCl}, 2.5 \mathrm{mM} \mathrm{CaCl}, \mathrm{pH}$ 7.4. The cell suspension was treated with $1 \mu \mathrm{M}$ Sytox Blue nucleic acid stain (Invitrogen) for $10 \mathrm{~min}$ at room temperature in the dark. The cell suspension was then analysed for SYTOX blue positive cells after gating for cells positive for mCherry fluorescence, using a BD FACS Canto ${ }^{\mathrm{TM}}$ II flow cytometer (BD Biosciences).

\section{Statistics}

Data are presented as mean value \pm standard error of the mean (SEM). Statistical comparisons between group means were performed using GraphPad Prism 6 software (Graph Pad software, Inc.). One-way or two-way ANOVA followed by post hoc Tukey test for multiple comparisons was used when justified. The significance threshold was set at $p=0.05$.

\section{Results}

\section{ALS/FTD mutant Cyclin F inhibits ER-Golgi transport}

To probe cellular disease mechanisms induced by mutant cyclin F, we first expressed familial ALS/FTDassociated mutant cyclin F (Fig. 1A) [3], tagged with mCherry, in HEK293T cells. Western blotting of cellular lysates prepared from cells expressing mutant cyclin $\mathrm{F}$ demonstrated no significant difference ( $p$ $>0.05$ ) between the expression levels of wild-type (WT) and mutant cyclin F (Fig. 1B, C).

Protein trafficking defects have been previously detected in ALS but have not been described before in association with mutant cyclin $\mathrm{F}[23,31]$. Hence, we first examined if mutant cyclin $\mathrm{F}$ inhibits ER-Golgi trafficking. We used a temperature sensitive mutant of vesicular stomatitis viral glycoprotein (VSVG ${ }^{\text {ts045) }}$ $[32,33,23,34]$, to examine trafficking of VSVG ${ }^{\text {ts } 045}$ from the ER to Golgi in cells co-expressing cyclin $F$ and VSVG ${ }^{\text {ts045 }}$ (Fig. 1D). VSVG ${ }^{\text {ts045 }}$ has been widely used as a reporter of membrane trafficking for canonical ER-to-Golgi transport $[35,36]$. The transport of VSVG ${ }^{\text {ts045 }}$ was examined by immunocytochemistry using anti-calnexin (Fig. 1E) or anti-GM130 (Fig. 1G) antibodies to label the ER and Golgi compartments, respectively. Analysis using Mander's coefficient, where 0 indicated no colocalisation and 1, high co-localisation, demonstrated that VSVG ${ }^{\mathrm{ts} 045}$ co-localised significantly more with calnexin in mutant cyclin $\mathrm{F}^{\mathrm{S} 621 \mathrm{G}}$ expressing cells $(0.6 \pm 0.03)$, after incubation at the permissive temperature $\left(32^{\circ} \mathrm{C}\right)$, compared to control cells expressing either cyclin $\mathrm{F}^{\mathrm{WT}}(0.3 \pm 0.03)$ or empty vector (EV) (expressing mCherry alone) $(0.06 \pm 0.009)$ (Fig. 1F). These data therefore indicate that more VSVG ${ }^{\text {ts } 045}$ was retained in the ER in mutant cyclin $\mathrm{F}^{\mathrm{S} 621 \mathrm{G}}$ expressing cells compared to controls. Additional control cells from each group were incubated only at $40^{\circ} \mathrm{C}$, confirming that VSVG misfolds and is retained within the ER at $40^{\circ} \mathrm{C}$ (Fig. $1 \mathrm{~F}$ ). Similarly, in cells expressing mutant cyclin $\mathrm{F}^{\mathrm{S} 621 \mathrm{G}}$, significantly less VSVG ${ }^{\text {ts045 }}$ colocalised with GM130 (0.2 \pm 0.02$)$ compared to control cells expressing EV $(0.8 \pm 0.02)$ or cyclin $\mathrm{F}^{\mathrm{WT}}(0.6 \pm 0.03)$ (Fig. $\left.1 \mathrm{H}\right)$, where VSVG ${ }^{\mathrm{ts} 045}$ extensively colocalised with GM130, indicating efficient ER-Golgi transport. Hence, VSVG ${ }^{\text {t5045 }}$ is retained in the ER and less is transported to the Golgi 
apparatus in cells expressing mutant cyclin $\mathrm{F}$ compared to control cells, indicating that mutant cyclin $\mathrm{F}$ perturbs ER-Golgi transport.

\section{ALS/FTD mutant cyclin F interferes with the budding of COPII vesicles from the ER}

ER-Golgi trafficking includes multiple steps, consisting of COPII-dependent vesicle budding from the ER, tethering and docking to target membranes, and finally SNARE protein-dependent vesicle fusion $[18,19]$. Newly synthesized proteins are exported from the ER via coat protein complex II (COPII)-coated vesicles, which form in specialized zones within the ER, termed ER exit sites (ERES) $[16,18,19]$. The first stage of ER-Golgi transport is therefore the formation of COPII vesicles from the ER membranes.

First, to provide evidence that VSVG ${ }^{\text {ts045 }}$ is transported from ER to Golgi in COPII vesicles, we repeated the experiment above (Fig. 1), but performed immunocytochemistry for Sec31A (rather than calnexin or GM130), a marker of the newly budded ER-derived vesicles at ER exit sites (Fig. 2A). Neuronal SH-SY5Y cells expressing GFP-tagged VSVG ${ }^{\text {ts } 045}$ were incubated overnight at $40^{\circ} \mathrm{C}$ to accumulate misfolded VSVG ${ }^{\text {ts } 045}$ followed by 30 min incubation at $32^{\circ} \mathrm{C}$. Following immunocytochemistry, VSVG ${ }^{\text {ts } 045}$ was found to be associated with Sec31-positive budding vesicles (Fig. 2B), consistent with its packaging into COPII vesicles to be transported from the ER.

We next used a modified in vitro reconstituted assay (Xu and Hay, 2004) to directly assess whether

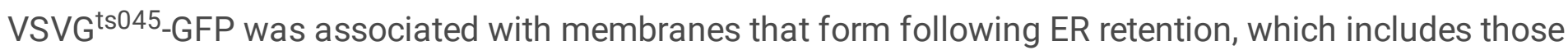
from COPII vesicles, the ER-Golgi intermediate compartment (ERGIC) and intra/post Golgi carriers (Fig. 2B). This assay therefore aims to reconstitute ER vesicle budding using rat liver cytosol (as a source of soluble COPII) and an ATP regeneration system. ER-derived COPII vesicles are released into the extracellular buffer during incubation [29, 37] and semi-intact cells are used as a source of ER, given that the integrity of the ER is preserved under these conditions [29, 38, 39]. Semi-intact HEK293T cells coexpressing cyclin $\mathrm{F}$ and VSVG ${ }^{\mathrm{ts} 045}$ for $24 \mathrm{~h}$ were incubated at $40^{\circ} \mathrm{C}$ overnight to retain VSVG ${ }^{\mathrm{ts} 045}$ in the ER, then incubated at $32^{\circ} \mathrm{C}$ to allow VSVG ${ }^{\text {ts } 045}$ to be incorporated into COPIl vesicles. The light membranes, including ER-derived vesicles, were then recovered by cellular fractionation (Fig. 2A).

VSVG ${ }^{\text {ts045 }}$ and Sec23 expression were first examined in control cell lysates sampled before the budding reaction. No significant difference in VSVG and Sec23 levels was detected between all groups (untreated cells or cells expressing EV, WT or mutant cyclin F) (Fig. 2C-E). This indicates that differences in protein expression do not account for the results of the budding assay.

VSVG ${ }^{\text {ts } 045}$ was then quantitated in the budded vesicular preparations by Western blotting using an antiVSVG antibody (Fig. 2F). Significantly less VSVG ${ }^{\text {ts } 045}$ was present in the preparations derived from cells expressing mutant cyclin $\mathrm{F}^{\mathrm{S} 621 \mathrm{G}}$ compared to UT cells (4.9-fold) and cells expressing mCherry only (EV) (3.8-fold, Fig. 2G), consistent with impairment in protein transport from the ER to Golgi (Fig. 1). There was a trend towards less VSVG ${ }^{\mathrm{ts} 045}$ in preparations derived from cells expressing mutant cyclin $\mathrm{F}^{\mathrm{S} 195 \mathrm{R}}$, but 
this was not statistically significant. The presence of COPII was also assessed in the vesicular fractions using anti-Sec23 antibodies (Fig. 2F). Interestingly, significantly less Sec23 was also associated with vesicles obtained from cells expressing mutant cyclin $\mathrm{F}^{\mathrm{S} 621 \mathrm{G}}$ or mutant cyclin $\mathrm{F}^{\mathrm{S} 195 \mathrm{R}}$ compared to UT

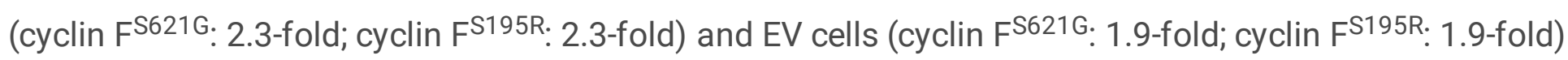
(Fig. 2H). These results reveal that less COPII and vesicular cargo (VSVG ${ }^{\text {ts045}}$ ) are present in ER-derived vesicular preparations from cells expressing mutant cyclin $\mathrm{F}$ compared to controls. This further implies that vesicular budding between the ER and Golgi is defective in cells expressing mutant cyclin F.

\section{ER-derived exit sites are perturbed in cells expressing ALS/FTD mutant cyclin F}

We next examined the first step of ER-Golgi trafficking by analysing Sec31-positive clusters of ER exit sites in HEK 293T cells expressing mutant cyclin F by airyscan microscopy (Fig. 3A-C). ER-exit site clusters were identified as hollow and spherical structures staining positive for Sec31, with a diameter ranging from 60 to $350 \mathrm{~nm}$. The mean diameter of these clusters was significantly decreased (1.2-fold, $p$ $<0.05$ ) in cells expressing ALS/FTD mutant cyclin F compared to UT cells or those expressing either EV or WT cyclin F (Fig. 3B). Significantly more small ER-exit site clusters and conversely, significantly fewer large ER-exit site clusters were present in cells expressing mutant cyclin F compared to UT, EV or WT cyclin F cells (Fig. 3C). These results suggest that the first step of ER-Golgi trafficking, vesicle budding from the ER, was perturbed in cells expressing ALS/FTD-mutant cyclin F.

It has been previously established that ubiquitination of Sec31 regulates the size of COPIl coats, allowing for the trafficking of large cargo [40], and we previously demonstrated that mutant cyclin F impairs ubiquitination [12]. This raises the possibility that aberrant ubiquitination of Sec31 in cells expressing ALS/FTD mutant cyclin F impacts the size of COPIl vesicles, perturbing the transport of large cargoes. The ubiquitination of Sec31 was therefore examined by immunoprecipitation (IP) in cells co-expressing FLAG-tagged Sec31 with either EV or cyclin F proteins (Fig. 3D). IP of cell lysates was performed using an anti-FLAG antibody, and Western blotting for ubiquitin was performed. The ubiquitination of Sec31 was significantly decreased in cells expressing mutant cyclin $\mathrm{F}^{\mathrm{S} 621 \mathrm{G}}$ compared to those expressing mCherry alone (EV, 5-fold) or cyclin $\mathrm{F}^{\mathrm{WT}}$ (8-fold) (Fig. 3E). These findings were not due to differences in the expression levels of Sec31 between populations (Fig. 3F, G). Hence, the ubiquitination of Sec31 was significantly decreased in cells expressing mutant cyclin $\mathrm{F}$.

To further examine the transport of large cargoes, bulk protein secretion was examined in the conditioned medium from cells expressing cyclin $\mathrm{F}$ (Fig. 3E). Silver staining revealed no significant difference in the levels of secreted proteins overall in the media. However, when proteins over $100 \mathrm{kDa}$ were specifically examined, significantly less total protein of this size was detected in the conditioned medium from mutant cyclin F expressing cells compared to the other groups (Fig. 3G). These results therefore imply that bulk secretion of large proteins is impaired in cells expressing mutant cyclin $F$, providing further evidence that mutant cyclin F perturbs protein trafficking between the ER and Golgi compartments. 


\section{ALS/FTD mutant cyclin F impedes ER-associated degradation}

The results described above demonstrate that one important function of the ER, the transport of secretory and transmembrane proteins from the ER to the Golgi apparatus, is perturbed by mutant cyclin F. ERAD is another important quality control function of the ER that monitors the fidelity of protein folding, and those proteins that fail to fold or assemble properly are degraded [41, 42]. Furthermore, ERAD is a complex, multistep process, that is regulated by ubiquitination, which is defective in cells expressing mutant cyclin F. Hence, we next examined whether ERAD is defective in cyclin F-associated ALS/FTD. ERAD begins with the recognition and targeting of substrates, followed by ubiquitination, retro-translocation and

proteasomal degradation. Here we used a substrate with an ER-targeted signal sequence ( $\mathrm{K}^{\mathrm{b}}$-SS) fused to a mutant version of Venus, ddVenus (deglycosylation-dependant Venus), in which Asp is substituted to Asn at position 82 [26]. This mutation results in glycosylation and a sharp reduction in fluorescence, which is restored to wildtype levels when Asn is converted back to Asp. Removal of oligosaccharides by endogenous peptide:N'glycanase (PNGase) in the cytosol results in deamidation of glycosylated Asn, converting it to Asp which restores fluorescence [26]. Given that ERAD involves two stages, entry of substrate into the ER followed by its retro-translocation to the cytoplasm, both glycosylation of ddVenus in the ER and its deglycosylation in the cytosol are required for fluorescence (Fig. 4A, B). Hence the accumulation of ddVenus fluorescence indicates specific impairment of ERAD. In addition, a second ERAD substrate was used, fluorescent ddVenus fused to the null Hong Kong genetic variant of a1antitrypsin (NHK-ddVenus), which misfolds terminally in the ER and is also degraded specifically by ERAD $[25,26,43]$. In cells expressing empty vector, cyclin $F^{W T}$ or mutant cyclin $F^{S 621 G}$, ERAD was probed using substrates NHK-Venus (a control for transfection efficiency), NHK-ddVenus or SS-ddVenus (Fig. 4C-E). Quantification demonstrated that significantly more fluorescent cells were present in mutant cyclin $\mathrm{F}$ expressing populations compared to cyclin FWT (NHK-ddVenus: 1.7-fold; SS-ddVenus: 1.5-fold,) or mCherry only (EV) populations (NHK-ddVenus: 33.1-fold; SS-ddVenus: 33.8-fold) (Fig. 3D-F). Hence, expression of mutant cyclin F significantly impairs ERAD compared to WT and controls.

\section{ALS/FTD cyclin F mutants induce ER stress}

The impairment of two important ER functions, protein trafficking to the Golgi and ERAD, implies that mutant cyclin $\mathrm{F}$ perturbs the overall homeostasis of the ER. To investigate this possibility further, we next examined whether cyclin $\mathrm{F}$ induces ER stress using immunocytochemistry following established methods [44-46] (Fig. 5). XBP-1 and CHOP are both transcription factors that translocate to the nucleus when activated during ER stress, and CHOP becomes activated only when the UPR becomes pro-apoptotic [47]. Nuclear immunoreactivity to XBP-1 and pro-apoptotic CHOP therefore indicate activation of the UPR (Fig. 5A). Expression of mutant cyclin F significantly increased the proportion of cells with nuclear XBP1

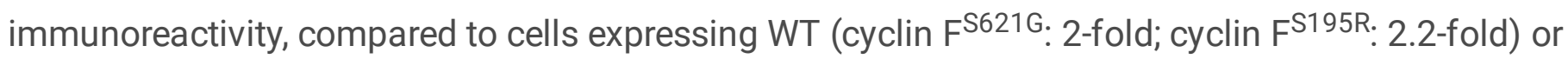
mCherry only (EV) (cyclin F ${ }^{S 621 G}$ : 5.9-fold; cyclin F ${ }^{S 195 R}$ : 6.3-fold) (Fig. 5B, C). Similarly, significantly more cells with nuclear immunoreactivity to $\mathrm{CHOP}$ were observed in populations expressing mutant cyclin $\mathrm{F}$ 
compared to cells expressing WT (S621G: 1.7-fold; S195R: 1.9-fold) or EV (cyclin F ${ }^{\text {S621G: }}$ 5.9-fold; cyclin F S195R: 6.6-fold; Fig. 5D, E). To note, compared to mCherry, expression of WT cyclin F resulted in significantly more cells with nuclear immunoreactivity to $\mathrm{CHOP}$, but not to XBP1. These results indicate that ALS/FTD-associated mutations in cyclin F induce ER stress in neuronal cells.

To confirm the findings obtained in cell lines, ER stress was next examined in primary cortical neurons expressing cyclin $\mathrm{F}$ by immunocytochemistry, where nuclear reactivity to $\mathrm{CHOP}$ indicated activation of the UPR (Fig. 5F). Quantification demonstrated that significantly more primary neurons expressing mutant cyclin F displayed nuclear immunoreactivity to CHOP (S621 G: 1.7-fold; S195R: 2.2-fold) compared to WT or mCherry (EV) cells (Fig. 5G). Hence, ALS/FTD-associated mutations in cyclin F activate ER stress in primary cortical neurons, confirming the results obtained in cell lines.

\section{ALS/FTD mutant cyclin F induces fragmentation of the Golgi}

Fragmentation of the Golgi apparatus results following induction of cellular stress, including ER and oxidative stress [48, 49], and when ER-Golgi trafficking is impaired [23, 50-52]. Hence, perturbation of ERGolgi transport and induction of ER stress by mutant cyclin F implies that Golgi fragmentation is also induced. Therefore, we next assessed whether the Golgi apparatus was fragmented in cells expressing mutant cyclin F (Fig. 6). The morphology of the Golgi was examined by immunocytochemistry using an anti-GM130 antibody. In SH-SY5Y (Fig. 6A) and HEK293T (Supplementary Fig. 2A) cells expressing EV or Cyclin $\mathrm{F}^{\mathrm{WT}}$, the Golgi displayed its typical morphology of continuous stacked membranous vesicles. However, in mutant cyclin $\mathrm{F}^{\mathrm{S} 621 \mathrm{G}}$ and cyclin $\mathrm{F}^{\mathrm{S} 195 \mathrm{R}}$ expressing HEK293T cells and SH-SY5Y cells, the Golgi apparatus was fragmented, displaying multiple disconnected elements or tubular-vesicular clusters [53]. Quantification revealed that the percentage of cells displaying fragmented Golgi was significantly increased in cellular populations expressing mutant cyclin F F $^{\text {621G }}$ (HEK293T: 1.5-fold; SH-SY5Y: 2-fold) or

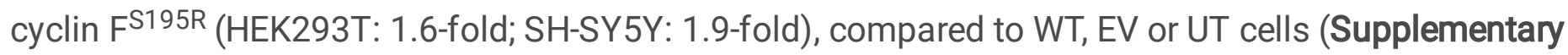
Fig. 2B and Fig. 6B). We also examined Golgi fragmentation in the GM130-immunostained HEK293 cells prepared for the VSVG ${ }^{\text {ts045 }}$ assay (Fig. 1). Quantification revealed that significantly more mutant cyclin $\mathrm{F}^{\mathrm{S} 621 \mathrm{G}}$ cells displayed fragmented Golgi compared to WT (2.6-fold) or EV cells (13.2-fold change, Supplementary Fig. $3 \mathrm{~A}$ ) to a similar degree in each case $(57.3 \pm 8.6 \%$, compared to $64.6 \pm 1 \%$ in Fig. 6$)$. Hence Golgi fragmentation was present in cells in which VSVG ${ }^{\mathrm{ts} 045}$ secretion was impaired, confirming that inhibition of ER-Golgi secretion correlates with fragmentation of the Golgi.

To further confirm these results and to provide a more unbiased quantification method, the surface area covered by the Golgi fragments was next assessed. Golgi stacks can be dispersed (mini-stacks) or completely disassembled, hence the surface area covered by the Golgi also indicates fragmentation [48]. Quantification of this area revealed a significant increase in the area covered by fragmented Golgi in cells expressing cyclin $\mathrm{F}^{\mathrm{S} 621 \mathrm{G}}$ (1.9-fold) and cyclin $\mathrm{F}^{\mathrm{S} 195 \mathrm{R}}$ (2.2-fold) mutants compared to cyclin $\mathrm{F}^{\mathrm{WT}}$ expressing cells and other controls (Fig. 6C). Similarly, we also examined the area covered by Golgi 
fragments in the GM130-immunostained HEK293 cells prepared for the VSVG ${ }^{\text {t5045 }}$ assay (Fig. 1). Quantification revealed that a significant increase in the area covered by fragmented Golgi was present in cells expressing cyclin $\mathrm{F}^{\mathrm{S} 621 \mathrm{G}}$ compared to WT (2.6-fold) or EV cells (13.5-fold, Supplementary Fig. 3b) again to a similar degree $(57.4 \%$, compared to $64.7 \%$ in Fig. 6). Hence inhibition of ER-Golgi secretion correlates with fragmentation of the Golgi. Together these results demonstrate that mutant cyclin $\mathrm{F}$ induces Golgi fragmentation in neuronal cells.

To further confirm the above results, we next examined mouse cortical primary neurons expressing mutant cyclin $\mathrm{F}$ and controls for Golgi fragmentation. Immunocytochemistry of primary neurons expressing cyclin $\mathrm{F}$ was first performed using an anti-GM130 antibody to examine the morphology of the Golgi apparatus (Fig. 6D). Significantly more neurons expressing mutant cyclin F displayed Golgi fragmentation compared to those expressing WT (cyclin $F^{\text {S621G. }}$ 2.2-fold; cyclin $\mathrm{F}^{\mathrm{S} 195 \mathrm{R}}$ : 2.9-fold) and

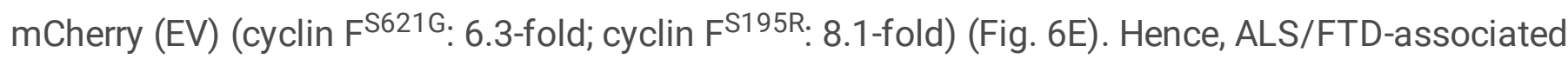
mutations in cyclin $\mathrm{F}$ induce Golgi fragmentation in primary cortical neurons, confirming the results obtained in cell lines.

\section{ALS/FTD mutant cyclin F induces cell death}

ER stress induces apoptosis when prolonged or severe, and Golgi fragmentation is also associated with apoptosis $[54,55]$. Hence cellular death was next analysed by flow cytometry following Sytox Blue staining, in SH-SY5Y cells expressing cyclin F (Fig. 7A, B). Quantitative analysis demonstrated significantly more dead cells in populations expressing mutant cyclin $\mathrm{F}^{\mathrm{S} 621 \mathrm{G}}$ compared to UT cells (3.7fold) and those expressing cyclin $\mathrm{F}^{\mathrm{WT}}$ (1.4-fold) or mCherry (3.8-fold), indicating that mutant cyclin $\mathrm{F}^{\mathrm{S} 621 \mathrm{G}}$ expression induces cell death.

This was next examined in primary neurons, where the presence of condensed nuclear morphology indicated the induction of apoptosis, as previous $[44,56]$. Examination of the nuclei and quantitative analysis of primary neurons expressing cyclin $\mathrm{F}$ demonstrated that the percentage of neurons undergoing apoptosis was significantly increased in populations expressing mutant cyclin $\mathrm{F}$ compared to those expressing cyclin $\mathrm{F}^{\mathrm{WT}}$ (cyclin $\mathrm{F}^{\mathrm{S} 621 \mathrm{G}}$ : 1.9-fold; cyclin $\mathrm{F}^{\mathrm{S} 195 \mathrm{R}}$ : 1.8-fold) or mCherry (cyclin $\mathrm{F}^{\mathrm{S} 621 \mathrm{G}}$ : 7.9-fold; cyclin F $195 R$ : 7.3-fold) (Fig. 7C, D). Hence, these data confirm that ALS/FTD-cyclin F mutants induce apoptosis.

\section{Discussion}

This study demonstrates that ALS/FTD-associated mutations in cyclin F perturb ER homeostasis by inhibiting two key functions; ERAD and transport of proteins from the ER to the Golgi apparatus. Consistent with these observations, Golgi fragmentation, ER stress, and induction of apoptosis was also detected in cell lines and mouse cortical primary neurons expressing mutant cyclin F. Together these data imply that dysfunction to the ER is an important process in neuronal degeneration induced by mutant cyclin F, thus providing novel insights into CCNF-associated ALS/FTD. 
ER-Golgi transport is a vital gateway to the endomembrane system because one third of all proteins transit through the ER-Golgi compartments before reaching their final cellular locations $[18,57]$. Our results imply that ALS/FTD-associated p.S621G and p.S195R mutations in cyclin F perturb the first stage of ER-Golgi transport: the budding of COPII vesicles from the ER. The COPII coat is composed of five separate proteins, including $\operatorname{Sec} 23$ and Sec31, and its assembly is essential for the formation of transport vesicles on the cytosolic face of the ER membrane. Curvature of the ER membrane, concentration of cargo and vesicular release then results. Defective COPII vesicles are known to inhibit secretion [40] and here we detected several abnormalities in COPII in mutant cyclin F cells. Less COPII and less vesicular cargo were present in ER-derived membranes obtained from mutant cyclin $\mathrm{F}$ expressing cells compared to controls. Furthermore, the Sec31-clusters (representing one or more ER-exit sites) were smaller in these cells, implying that either fewer COPII vesicles were formed overall or that the same number of COPII vesicles were present, but each contained less Sec31. Typical COPII vesicles are 60-70 nm in diameter, and it was not possible to resolve individual COPII vesicles or ER-exit sites using the methods used here. Furthermore, whilst Sec23 levels correlate with the load of protein cargo [40], here the levels of Sec23 were comparable to cargo load, suggesting that the defect is related to aberrant COPII vesicles themselves rather than the incorporation of protein cargo. Hence, these results imply that there is a defect in the formation of COPII vesicles and/or ER-exit sites in mutant cyclin F expressing cells.

COPII vesicles are normally too small to transport some secreted macromolecules, including procollagen fibrils, pre-chylomicrons, and pre- very low-density lipoproteins (VLDLs). However, ubiquitination of Sec31 by the CUL3-KLHL12 complex allows for the generation of large vesicles to facilitate the transport of these bulky cargo, thus controlling the traffic of COPII vesicles [40,58]. Calcium-dependant control of CUL3-KLHL12 also ubiquitinates lysine residues on Sec31 [40,59]. Similarly, deubiquitylation of Sec31 by USP8 antagonizes the formation of large procollagen-containing carriers [60]. The exact site of Sec31 ubiquitination may not be crucial for regulation of COPII vesicle trafficking, but it is a signal to recruit other effectors to assemble the coat or to regulate its catalytic activity. We previously demonstrated that mutant cyclin $\mathrm{F}^{\mathrm{S} 621 \mathrm{G}}$ dysregulates ubiquitination at Lys48, resulting in disruption of biological networks responsive for cellular survival and maintenance $[11,13]$. Here, we demonstrate that in cells expressing mutant cyclin F, ubiquitination of Sec31 was reduced and bulk secretion of large proteins ( $>100 \mathrm{kDa}$ ) was inhibited, although the secretion of proteins smaller than this was unchanged. This together with inhibition of ER-Golgi trafficking in these cells implies that aberrant ubiquitination of Sec31 impairs the formation of COPII vesicles, forming smaller ER-exit site clusters and inhibiting the transport of large cargoes, which would ultimately impact neuronal functions. Future studies are therefore warranted to confirm this possibility and to identify the specific large cargoes whose secretion is inhibited by mutant cyclin F. Mutations in the genes encoding Sec23A [61] or Sar1B [62] lead to the accumulation of large proteins such as collagen, or lipid particles into the ER [61] which causes Skull-Lenticular-Sutural Dysplasia [61] and Chylomicron Retention Disease [62]. This highlights the importance of the integrity of COPII vesicles in the regulation of protein secretion. 
Collagen forms the major structural component of the extracellular matrix and it is the most abundant cargo for COPII vesicles [60]. This is not surprising given that collagen constitutes up to $30 \%$ of the total protein mass of a typical organism. It was previously believed that fibrillar collagens are absent in the brain [63]. However, neurons are now known to express collagen [64, 65], and an increasing number of studies highlight key roles for collagen in both the PNS and CNS [66]. Whilst this area remains poorly understood, it is clear that expression and deposition of collagen in the nervous system is highly dynamic and tightly controlled, and is implicated in regulation of axonal outgrowth and synaptic differentiation [67]. Furthermore, some types of collagen are regulated in response to stress and are dysregulation in several neurological conditions, including Alzheimer's diseases[66]. regulated in conditions. COL6

Ubiquitination also regulates ERAD because poly-ubiquitin chains on ERAD substrates are remodelled at several stages during ERAD $[68,69]$. Interestingly, we also detected impairment of ERAD in cells expressing mutant cyclin F. ERAD normally removes terminally misfolded proteins from the ER lumen or membrane, targeting them for degradation to membrane-embedded E3 ligase complexes, where they undergo ubiquitination on their cytosolically exposed protein domains. Hence, misfolded proteins in the ER would not be degraded efficiently in cyclin F-associated ALS/FTD, leading to their accumulation in the ER. This would further induce ER stress and disrupt ER homeostasis. These mechanisms may all combine, thus exacerbating ER stress and further perturbing ER homeostasis. Consistent with activation of pro-apoptotic CHOP during ER stress, we also demonstrate that expression of ALS/FTD cyclin F mutants induces neuronal death. This finding is also consistent with apoptotic death observed in the transient fish models overexpressing S621G cyclin F [13].

Impairment of ER-Golgi and other forms of transport has been previously described in ALS [23, 70-73], in cells expressing mutant SOD1 [23], mutant TDP-43 [23], mutant FUS [23] or mutant ubiquilin2 [31], and these events were previously linked to ER stress [23]. ER stress has now been widely implicated in ALS and mutations in SOD1 [44], TARDBP [74], FUS [75], OPTN [76-78], VCP [79], UBQLN2 [31], VAPB [80, 81] and C9orf72 [82] all induce ER stress. Previously it has been established that mutant forms of SOD1 and TDP-43 induce ER stress from the cytoplasm [70, 71, 83], or from the cytoplasmic face of the ER [23] respectively, by inhibiting ER-Golgi transport [23]. Preliminary experiments examining whether cyclin $\mathrm{F}$ is present in the ER were inconclusive, raising the question of how ER stress is induced in ALS/FTD. As ALS mutant cyclin F mislocalizes to the cytoplasm where it promotes cytoplasmic aggregation of TDP-43 [84], it is possible that mutant cyclin F induces ER stress from the cytoplasm by inhibiting ER-Golgi transport. However, we cannot rule out the possibility that mutant cyclin F may directly induce ER stress from the ER itself, although mutant cyclin $\mathrm{F}$ aberrantly ubiquitinates $\mathrm{Sec} 31$, which is also localised in the cytoplasm.

Golgi fragmentation is also a well-described event in $\operatorname{ALS}[24,53,85]$ where the Golgi apparatus undergoes morphological changes, resulting in disruption of its characteristic ribbon-like structure [85, 86]. Fragmentation of the Golgi apparatus has been described in sporadic ALS patients [87] and before disease onset in SOD1 ${ }^{\mathrm{G} 93 \mathrm{~A}}$ mice [88], prior to neuromuscular denervation and axon retraction [89]. It is also a feature of other neurodegenerative diseases [85] but it has not been previously described for mutant cyclin F. In this study, we demonstrate that ALS/FTD-associated mutations in cyclin F trigger 
Golgi fragmentation. The proper organization of the Golgi depends on efficient bidirectional ER-Golgi vesicle transport $[86,90]$ and both membrane flow and cargo load influence its structure and function. Hence, blocking the export of cargo-containing ER carriers [91, 92] or depleting cargo receptors [93] results in Golgi fragmentation and some of the tubulo-vesicular Golgi clusters can further fuse with the ER, increasing ER stress [94]. In addition, inhibition of intra-Golgi trafficking or vesicle transport from the Golgi to the plasma membrane can result in Golgi fragmentation, if prolonged [50-52]. Hence, in this study, it is possible that the inhibition of ER-Golgi transport by mutant cyclin $\mathrm{F}$ triggers Golgi fragmentation and ER stress. However, there are many possible cellular stressors than induce Golgi fragmentation so the directionality of these links cannot be conclusively established.

\section{Conclusion}

In summary, this study identifies novel cellular mechanisms triggered by ALS/FTD-associated mutant cyclin F. In Fig. 8, we provide one hypothetical model to illustrate the cellular events triggered by mutant cyclin F, based on the findings of this study. However, it is also possible that ER stress or Golgi fragmentation is the upstream trigger, which would subsequently trigger impairment of ER-Golgi transport and impede ERAD given that these events are all closely related. Alternatively, it is possible that the cellular events detected in this study result from a combination of these defects. Further studies are therefore required to probe the directionality of these links.

\section{Abbreviations}

\section{ALS}

Amyotrophic lateral sclerosis

BSA

Bovine serum albumin

CUL1

cullin-1

COPII

Coat protein complex II

ER

Endoplasmic reticulum

ERAD

Endoplasmic reticulum associated degradation

ERES

ER exit sites

EV

Empty vector

FALS

Familial ALS 
FTD

Frontotemporal dementia

NLS

Nuclear localization signals

PFA

Paraformaldehyde

SALS

Sporadic ALS

SKP1

S-phase kinase-associated protein 1

SEM

standard error of the mean

UPR

Unfolded protein response

UPS

Ubiquitin proteasome system

UT

untransfected

VSVG

vesicular stomatitis viral glycoprotein

WT

Wild-type

\section{Declarations}

\section{Ethical Approval}

All husbandry and experimental procedures were performed in compliance with the Animal Ethics Committee, Macquarie University, NSW, Australia (ARA 2017/030) and the Internal Biosafety Committee, Macquarie University (NLRD 5201401007 and 5974-52019597412350).

\section{Consent to participate}

Not applicable

\section{Consent for publication}

All authors discussed results, provided feedback, and approved the final manuscript. 


\section{Availability of data and material}

The datasets generated during and analysed during the current study are available from the corresponding author on reasonable request.

\section{Competing interests}

The authors declare that they have no competing financial interests.

\section{Funding}

This work was supported by grants from the National Health and Medical Research Council of Australia (NHMRC) [10305133, 1086887, 1095215].

\section{Author contributions}

J.D.A: conceived and directed the project. A.M.G.R., V.S. and J.D.A.: designed the experiments. A.M.G.R. and V.S.: performed the VSVG assay; A.M.G.R. analysed the results; A.M.G.R.: performed and analysed budding assay with the help of M.B.; A.M.G.R.: performed and analysed immunoprecipitation experiments with the help of S.L.R. and A.L.; A.M.G.R.: performed silver stain of bulk proteins with the help of M.V., C.J. and S.S.; A.M.G.R.: performed mouse neuronal primary cultures, transfection and immunohistochemistry with the help of A.K. A.M.G.R: performed and analysed ER stress, ERAD, Golgi fragmentation experiments. A.M.G.R. and V.S.: performed and analysed cell death experiments. A.M.G.R. and J.D.A: wrote the manuscript. A.M.G.R, L.O., K.L.W., S.Y., I.P.B., R.S.C., J.D.A.: revised the manuscript. All authors read and approved the final manuscript.

\section{Author information}

\section{Affiliations}

- Macquarie University, Centre for Motor Neuron Disease Research, Department of Biomedical Sciences, Faculty of Medicine, Health and Human Sciences, Macquarie University, Sydney, NSW 2109, Australia.

Audrey M.G. Ragagnin, Vinod Sundaramoorthy, Marta Vidal, Cyril J. Jagaraj, Sina Shadfar, Mariana Brocardo, Sonam Parakh, Anna Konopka, Natalie Grima, Shu Yang, Stephanie L. Rayner, Kelly L. Williams, Ian P. Blair, Roger S. Chung, Albert Lee, Julie D. Atkin

- Illawarra Health and Medical Research Institute, Wollongong, New South Wales, Australia. 
Lezanne Ooi

- School of Chemistry and Molecular Bioscience and Molecular Horizons, University of Wollongong, New South Wales, Australia.

Lezanne Ooi

- Department of Biochemistry and Genetics, La Trobe Institute for Molecular Science, La Trobe University, Bundoora, Melbourne, VIC 3086, Australia.

Julie D. Atkin

Corresponding authors

Correspondence and request for materials should be addressed to Julie D. Atkin (email: julie.atkin@mq.edu.au) or Audrey M.G. Ragagnin (audrey.ragagnin@riken.jp).

\section{Acknowledgements}

We thank Dr Jess E Grotzke for constructs encoding single strand ddVenus, ddVenus and null Hong-Kong substrate fused to Venus [26]. We thank Dr Lippincott-Schwartz for the VSVG ${ }^{\text {ts } 045}$ construct fused to eGFP.

\section{References}

1. DeJesus-Hernandez M, Mackenzie IR, Boeve BF, Boxer AL, Baker M, Rutherford NJ, et al. Expanded GGGGCC hexanucleotide repeat in noncoding region of C90RF72 causes chromosome 9p-linked FTD and ALS. Neuron. 2011;72:245-56.

2. Renton AE, Majounie E, Waite A, Simón-Sánchez J, Rollinson S, Gibbs JR, et al. A hexanucleotide repeat expansion in C9ORF72 is the cause of chromosome 9p21-linked ALS-FTD. Neuron. 2011;72:257-68.

3. Williams KL, Topp S, Yang S, Smith B, Fifita JA, Warraich ST, et al. CCNF mutations in amyotrophic lateral sclerosis and frontotemporal dementia. Nat Commun. 2016;7:11253.

4. D’Angiolella V, Esencay M, Pagano M. A cyclin without cyclin-dependent kinases: cyclin F controls genome stability through ubiquitin-mediated proteolysis. Trends Cell Biol. 2013;23:135-40.

5. Bai C, Richman R, Elledge SJ. Human cyclin F. EMBO J. 1994;13:6087-98.

6. D’Angiolella V, Donato V, Vijayakumar S, Saraf A, Florens L, Washburn MP, et al. SCF(Cyclin F) controls centrosome homeostasis and mitotic fidelity through CP110 degradation. Nature. 2010;466:138-42.

7. Skaar JR, Pagan JK, Pagano M. Mechanisms and function of substrate recruitment by F-box proteins. Nat Rev Mol Cell Biol. 2013;14:369-81. 
8. Fung TK, Siu WY, Yam CH, Lau A, Poon RYC. Cyclin F is degraded during G2-M by mechanisms fundamentally different from other cyclins. J Biol Chem. 2002;277:35140-9.

9. Hicke L, Dunn R. Regulation of membrane protein transport by ubiquitin and ubiquitin-binding proteins. Annu Rev Cell Dev Biol. 2003;19:141-72.

10. Rape M. Ubiquitylation at the crossroads of development and disease. Nat Rev Mol Cell Biol. 2018;19:59.

11. Lee A, Rayner SL, De Luca A, Gwee SSL, Morsch M, Sundaramoorthy V, et al. Casein kinase II phosphorylation of cyclin $\mathrm{F}$ at serine 621 regulates the Lys48-ubiquitylation E3 ligase activity of the SCF(cyclin F) complex. Open Biol. 2017;7:170058.

12. Lee A, Rayner SL, Gwee SSL, De Luca A, Shahheydari H, Sundaramoorthy V, et al. Pathogenic mutation in the ALS/FTD gene, CCNF, causes elevated Lys48-linked ubiquitylation and defective autophagy. Cell Mol Life Sci CMLS. 2018;75:335-54.

13. Hogan AL, Don EK, Rayner SL, Lee A, Laird AS, Watchon M, et al. Expression of ALS/FTD-linked mutant CCNF in zebrafish leads to increased cell death in the spinal cord and an aberrant motor phenotype. Hum Mol Genet. 2017;26:2616-26.

14. Webster CP, Smith EF, Shaw PJ, De Vos KJ. Protein Homeostasis in Amyotrophic Lateral Sclerosis: Therapeutic Opportunities? Front Mol Neurosci. 2017;10:123.

15. Shahheydari H, Ragagnin A, Walker AK, Toth RP, Vidal M, Jagaraj CJ, et al. Protein Quality Control and the Amyotrophic Lateral Sclerosis/Frontotemporal Dementia Continuum. Front Mol Neurosci. 2017;10:119.

16. Zeuschner D, Geerts WJ, van Donselaar E, Humbel BM, Slot JW, Koster AJ, et al. Immuno-electron tomography of ER exit sites reveals the existence of free COPII-coated transport carriers. Nat Cell Biol. 2006;8:377-83.

17. Aridor M, Bannykh SI, Rowe T, Balch WE. Sequential coupling between COPII and COPI vesicle coats in endoplasmic reticulum to Golgi transport. J Cell Biol. 1995;131:875-93.

18. McCaughey J, Stephens DJ. ER-to-Golgi Transport: A Sizeable Problem. Trends Cell Biol. 2019;29:940-53.

19. Gomez-Navarro N, Miller E. Protein sorting at the ER-Golgi interfaceProtein sorting in the ER and Golgi. J Cell Biol. 2016;215:769-78.

20. Nakatsukasa K, Brodsky JL. The Recognition and Retrotranslocation of Misfolded Proteins from the Endoplasmic Reticulum. Traffic Cph Den. 2008;9:861-70.

21. Peterson BG, Glaser ML, Rapoport TA, Baldridge RD. Cycles of autoubiquitination and deubiquitination regulate the ERAD ubiquitin ligase Hrd1. eLife. 8:e50903.

22. Mukherjee S, Chiu R, Leung S-M, Shields D. Fragmentation of the Golgi apparatus: an early apoptotic event independent of the cytoskeleton. Traffic Cph Den. 2007;8:369-78.

23. Soo KY, Halloran M, Sundaramoorthy V, Parakh S, Toth RP, Southam KA, et al. Rab1-dependent ERGolgi transport dysfunction is a common pathogenic mechanism in SOD1, TDP-43 and FUS- 
associated ALS. Acta Neuropathol (Berl). 2015;130:679-97.

24. Martínez-Menárguez J, Tomás M, Martínez-Martínez N, Martínez-Alonso E. Golgi Fragmentation in Neurodegenerative Diseases: Is There a Common Cause? Cells. 2019;8:748.

25. Sifers RN, Brashears-Macatee S, Kidd VJ, Muensch H, Woo SL. A frameshift mutation results in a truncated alpha 1-antitrypsin that is retained within the rough endoplasmic reticulum. J Biol Chem. 1988;263:7330-5.

26. Grotzke JE, Lu Q, Cresswell P. Deglycosylation-dependent fluorescent proteins provide unique tools for the study of ER-associated degradation. Proc Natl Acad Sci U S A. 2013;110:3393-8.

27. Hosie KA, King AE, Blizzard CA, Vickers JC, Dickson TC. Chronic excitotoxin-induced axon degeneration in a compartmented neuronal culture model. ASN Neuro. 2012;4.

28. Bolte $\mathrm{S}$, Cordelières FP. A guided tour into subcellular colocalization analysis in light microscopy. $J$ Microsc. 2006;224:213-32.

29. Xu D, Hay JC. Reconstitution of COPII vesicle fusion to generate a pre-Golgi intermediate compartment. J Cell Biol. 2004;167:997-1003.

30. Crowley L, Marfell B, Waterhouse N. Analyzing Cell Death by Nuclear Staining with Hoechst 33342. Cold Spring Harb Protoc. 2016;2016:778-81.

31. Halloran M, Ragagnin AMG, Vidal M, Parakh S, Yang S, Heng B, et al. Amyotrophic lateral sclerosislinked UBQLN2 mutants inhibit endoplasmic reticulum to Golgi transport, leading to Golgi fragmentation and ER stress. Cell Mol Life Sci CMLS. 2020;77:3859-73.

32. Presley JF, Cole NB, Schroer TA, Hirschberg K, Zaal KJ, Lippincott-Schwartz J. ER-to-Golgi transport visualized in living cells. Nature. 1997;389:81-5.

33. Nehls S, Snapp EL, Cole NB, Zaal KJ, Kenworthy AK, Roberts TH, et al. Dynamics and retention of misfolded proteins in native ER membranes. Nat Cell Biol. 2000;2:288-95.

34. Halloran M, Ragagnin AMG, Vidal M, Parakh S, Yang S, Heng B, et al. Amyotrophic lateral sclerosislinked UBQLN2 mutants inhibit endoplasmic reticulum to Golgi transport, leading to Golgi fragmentation and ER stress. Cell Mol Life Sci. 2020;77:3859-73.

35. Gallione CJ, Rose JK. A single amino acid substitution in a hydrophobic domain causes temperaturesensitive cell-surface transport of a mutant viral glycoprotein. J Virol. 1985;54:374-82.

36. McCaughey J, Stephens DJ. COPII-dependent ER export in animal cells: adaptation and control for diverse cargo. Histochem Cell Biol. 2018;150:119-31.

37. Joglekar AP, Xu D, Rigotti DJ, Fairman R, Hay JC. The SNARE motif contributes to rbet1 intracellular targeting and dynamics independently of SNARE interactions. J Biol Chem. 2003;278:14121-33.

38. Prosser DC, Tran D, Gougeon P-Y, Verly C, Ngsee JK. FFAT rescues VAPA-mediated inhibition of ER-toGolgi transport and VAPB-mediated ER aggregation. J Cell Sci. 2008;121:3052-61.

39. Shimoni Y, Schekman R. Vesicle budding from endoplasmic reticulum. Methods Enzymol. 2002;351:258-78. 
40. Jin L, Pahuja KB, Wickliffe KE, Gorur A, Baumgärtel C, Schekman R, et al. Ubiquitin-dependent regulation of COPII coat size and function. Nature. 2012;482:495-500.

41. Mehrtash $A B$, Hochstrasser M. Ubiquitin-dependent protein degradation at the endoplasmic reticulum and nuclear envelope. Semin Cell Dev Biol. 2019;93:111-24.

42. Wu X, Rapoport TA. Mechanistic insights into ER-associated protein degradation. Curr Opin Cell Biol. 2018;53:22-8.

43. Hosokawa N, Tremblay LO, You Z, Herscovics A, Wada I, Nagata K. Enhancement of endoplasmic reticulum (ER) degradation of misfolded Null Hong Kong alpha1-antitrypsin by human ER mannosidase I. J Biol Chem. 2003;278:26287-94.

44. Walker AK, Farg MA, Bye CR, McLean CA, Horne MK, Atkin JD. Protein disulphide isomerase protects against protein aggregation and is S-nitrosylated in amyotrophic lateral sclerosis. Brain. 2010;133:105-16.

45. Oslowski CM, Urano F. Measuring ER stress and the unfolded protein response using mammalian tissue culture system. Methods Enzymol. 2011;490:71-92.

46. Alam S, Abdullah CS, Aishwarya R, Orr AW, Traylor J, Miriyala S, et al. Sigmar1 regulates endoplasmic reticulum stress-induced C/EBP-homologous protein expression in cardiomyocytes. Biosci Rep. 2017;37.

47. Hu H, Tian M, Ding C, Yu S. The C/EBP Homologous Protein (CHOP) Transcription Factor Functions in Endoplasmic Reticulum Stress-Induced Apoptosis and Microbial Infection. Front Immunol. 2018;9:3083.

48. Machamer CE. The Golgi complex in stress and death. Front Neurosci. 2015;9:421.

49. Hicks SW, Machamer CE. Golgi structure in stress sensing and apoptosis. Biochim Biophys Acta. 2005;1744:406-14.

50. Persson R, Schnell CR, Borg LA, Fries E. Accumulation of Golgi-processed secretory proteins in an organelle of high density upon reduction of ATP concentration in rat hepatocytes. J Biol Chem. 1992;267:2760-6.

51. Zolov SN, Lupashin VV. Cog3p depletion blocks vesicle-mediated Golgi retrograde trafficking in HeLa cells. J Cell Biol. 2005;168:747-59.

52. Zhou Z, Mogensen MM, Powell PP, Curry S, Wileman T. Foot-and-mouth disease virus $3 \mathrm{C}$ protease induces fragmentation of the Golgi compartment and blocks intra-Golgi transport. J Virol. 2013;87:11721-9.

53. Haase G, Rabouille C. Golgi Fragmentation in ALS Motor Neurons. New Mechanisms Targeting Microtubules, Tethers, and Transport Vesicles. Front Neurosci. 2015;9:448.

54. Liu C, Mei M, Li Q, Roboti P, Pang Q, Ying Z, et al. Loss of the golgin GM130 causes Golgi disruption, Purkinje neuron loss, and ataxia in mice. Proc Natl Acad Sci U S A. 2017;114:346-51.

55. Eisenberg-Lerner A, Benyair R, Hizkiahou N, Nudel N, Maor R, Kramer MP, et al. Golgi organization is regulated by proteasomal degradation. Nat Commun. 2020;11:409. 
56. Crowley LC, Marfell BJ, Waterhouse NJ. Analyzing Cell Death by Nuclear Staining with Hoechst 33342. Cold Spring Harb Protoc. 2016;2016.

57. Brandizzi F. Transport from the endoplasmic reticulum to the Golgi in plants: Where are we now? Semin Cell Dev Biol. 2018;80:94-105.

58. Farhan $\mathrm{H}$, Weiss M, Tani K, Kaufman RJ, Hauri H-P. Adaptation of endoplasmic reticulum exit sites to acute and chronic increases in cargo load. EMBO J. 2008;27:2043-54.

59. McGourty CA, Akopian D, Walsh C, Gorur A, Werner A, Schekman R, et al. Regulation of the CUL3 Ubiquitin Ligase by a Calcium-Dependent Co-adaptor. Cell. 2016;167:525-38.e14.

60. Kawaguchi K, Endo A, Fukushima T, Madoka Y, Tanaka T, Komada M. Ubiquitin-specific protease 8 deubiquitinates Sec31A and decreases large COPII carriers and collagen IV secretion. Biochem Biophys Res Commun. 2018;499:635-41.

61. Boyadjiev SA, Fromme JC, Ben J, Chong SS, Nauta C, Hur DJ, et al. Cranio-lenticulo-sutural dysplasia is caused by a SEC23A mutation leading to abnormal endoplasmic-reticulum-to-Golgi trafficking. Nat Genet. 2006;38:1192-7.

62. Fryer LGD, Jones B, Duncan EJ, Hutchison CE, Ozkan T, Williams PA, et al. The endoplasmic reticulum coat protein II transport machinery coordinates cellular lipid secretion and cholesterol biosynthesis. J Biol Chem. 2014;289:4244-61.

63. Hubert T, Grimal S, Carroll P, Fichard-Carroll A. Collagens in the developing and diseased nervous system. Cell Mol Life Sci CMLS. 2009;66:1223-38.

64. Sund $M$, Väisänen T, Kaukinen $S$, llves $M, T u ~ H$, Autio-Harmainen $H$, et al. Distinct expression of type XIII collagen in neuronal structures and other tissues during mouse development. Matrix Biol J Int Soc Matrix Biol. 2001;20:215-31.

65. Su J, Gorse K, Ramirez F, Fox MA. Collagen XIX is expressed by interneurons and contributes to the formation of hippocampal synapses. J Comp Neurol. 2010;518:229-53.

66. Gregorio I, Braghetta P, Bonaldo P, Cescon M. Collagen VI in healthy and diseased nervous system. Dis Model Mech. 2018;11:dmm032946.

67. Fox MA, Sanes JR, Borza D-B, Eswarakumar VP, Fässler R, Hudson BG, et al. Distinct target-derived signals organize formation, maturation, and maintenance of motor nerve terminals. Cell. 2007;129:179-93.

68. Lopata A, Kniss A, Löhr F, Rogov VV, Dötsch V. Ubiquitination in the ERAD Process. Int J Mol Sci [Internet]. 2020 [cited 2021 Apr 19];21. Available from: https://www.ncbi.nlm.nih.gov/pmc/articles/PMC7432864/.

69. Preston GM, Brodsky JL. The evolving role of ubiquitin modification in endoplasmic reticulumassociated degradation. Biochem J. 2017;474:445-69.

70. Nishitoh H, Kadowaki H, Nagai A, Maruyama T, Yokota T, Fukutomi H, et al. ALS-linked mutant SOD1 induces ER stress- and ASK1-dependent motor neuron death by targeting Derlin-1. Genes Dev. 2008;22:1451-64. 
71. Atkin JD, Farg MA, Soo KY, Walker AK, Halloran M, Turner BJ, et al. Mutant SOD1 inhibits ER-Golgi transport in amyotrophic lateral sclerosis. J Neurochem. 2014;129:190-204.

72. Dascher $C$, Balch WE. Dominant inhibitory mutants of ARF1 block endoplasmic reticulum to Golgi transport and trigger disassembly of the Golgi apparatus. J Biol Chem. 1994;269:1437-48.

73. Wilson BS, Nuoffer C, Meinkoth JL, McCaffery M, Feramisco JR, Balch WE, et al. A Rab1 mutant affecting guanine nucleotide exchange promotes disassembly of the Golgi apparatus. J Cell Biol. 1994;125:557-71.

74. Walker AK, Soo KY, Sundaramoorthy V, Parakh S, Ma Y, Farg MA, et al. ALS-associated TDP-43 induces endoplasmic reticulum stress, which drives cytoplasmic TDP-43 accumulation and stress granule formation. PloS One. 2013;8:e81170.

75. Farg MA, Soo KY, Walker AK, Pham H, Orian J, Horne MK, et al. Mutant FUS induces endoplasmic reticulum stress in amyotrophic lateral sclerosis and interacts with protein disulfide-isomerase. Neurobiol Aging. 2012;33:2855-68.

76. Fifita JA, Williams KL, Sundaramoorthy V, Mccann EP, Nicholson GA, Atkin JD, et al. A novel amyotrophic lateral sclerosis mutation in OPTN induces ER stress and Golgi fragmentation in vitro. Amyotroph Lateral Scler Front Degener. 2017;18:126-33.

77. Sundaramoorthy V, Walker AK, Yerbury J, Soo KY, Farg MA, Hoang V, et al. Extracellular wildtype and mutant SOD1 induces ER-Golgi pathology characteristic of amyotrophic lateral sclerosis in neuronal cells. Cell Mol Life Sci CMLS. 2013;70:4181-95.

78. Deng H-X, Bigio EH, Zhai H, Fecto F, Ajroud K, Shi Y, et al. Differential involvement of optineurin in amyotrophic lateral sclerosis with or without SOD1 mutations. Arch Neurol. 2011;68:1057-61.

79. Gitcho MA, Strider J, Carter D, Taylor-Reinwald L, Forman MS, Goate AM, et al. VCP mutations causing frontotemporal lobar degeneration disrupt localization of TDP-43 and induce cell death. $J$ Biol Chem. 2009;284:12384-98.

80. Kanekura K, Nishimoto I, Aiso S, Matsuoka M. Characterization of amyotrophic lateral sclerosislinked P56S mutation of vesicle-associated membrane protein-associated protein B (VAPB/ALS8). J Biol Chem. 2006;281:30223-33.

81. Gkogkas C, Middleton S, Kremer AM, Wardrope C, Hannah M, Gillingwater TH, et al. VAPB interacts with and modulates the activity of ATF6. Hum Mol Genet. 2008;17:1517-26.

82. Zhang Y-J, Jansen-West K, Xu Y-F, Gendron TF, Bieniek KF, Lin W-L, et al. Aggregation-prone c9FTD/ALS poly(GA) RAN-translated proteins cause neurotoxicity by inducing ER stress. Acta Neuropathol (Berl). 2014;128:505-24.

83. Chang LY, Slot JW, Geuze HJ, Crapo JD. Molecular immunocytochemistry of the CuZn superoxide dismutase in rat hepatocytes. J Cell Biol. 1988;107:2169-79.

84. Yu Y, Nakagawa T, Morohoshi A, Nakagawa M, Ishida N, Suzuki N, et al. Pathogenic mutations in the ALS gene CCNF cause cytoplasmic mislocalization of Cyclin F and elevated VCP ATPase activity. Hum Mol Genet. 2019;28:3486-97. 
85. Sundaramoorthy V, Sultana JM, Atkin JD. Golgi fragmentation in amyotrophic lateral sclerosis, an overview of possible triggers and consequences. Front Neurosci. 2015;9:400.

86. Saraste J, Prydz K. A New Look at the Functional Organization of the Golgi Ribbon. Front Cell Dev Biol. 2019;7:171.

87. Fujita Y, Okamoto K, Sakurai A, Gonatas NK, Hirano A. Fragmentation of the Golgi apparatus of the anterior horn cells in patients with familial amyotrophic lateral sclerosis with SOD1 mutations and posterior column involvement. J Neurol Sci. 2000;174:137-40.

88. Stieber A, Gonatas JO, Collard J, Meier J, Julien J, Schweitzer P, et al. The neuronal Golgi apparatus is fragmented in transgenic mice expressing a mutant human SOD1, but not in mice expressing the human NF-H gene. J Neurol Sci. 2000;173:63-72.

89. van Dis V, Kuijpers M, Haasdijk ED, Teuling E, Oakes SA, Hoogenraad CC, et al. Golgi fragmentation precedes neuromuscular denervation and is associated with endosome abnormalities in SOD1-ALS mouse motor neurons. Acta Neuropathol Commun. 2014;2:38.

90. Lippincott-Schwartz J, Roberts TH, Hirschberg K. Secretory protein trafficking and organelle dynamics in living cells. Annu Rev Cell Dev Biol. 2000;16:557-89.

91. Storrie B, White J, Röttger S, Stelzer EH, Suganuma T, Nilsson T. Recycling of Golgi-resident glycosyltransferases through the ER reveals a novel pathway and provides an explanation for nocodazole-induced Golgi scattering. J Cell Biol. 1998;143:1505-21.

92. Marra P, Salvatore L, Mironov A Jr, Di Campli A, Di Tullio G, Trucco A, et al. The biogenesis of the Golgi ribbon: the roles of membrane input from the ER and of GM130. Mol Biol Cell. 2007;18:1595608.

93. Mitrovic S, Ben-Tekaya H, Koegler E, Gruenberg J, Hauri H-P. The cargo receptors Surf4, endoplasmic reticulum-Golgi intermediate compartment (ERGIC)-53, and p25 are required to maintain the architecture of ERGIC and Golgi. Mol Biol Cell. 2008;19:1976-90.

94. Puri S, Linstedt AD. Capacity of the Golgi apparatus for biogenesis from the endoplasmic reticulum. Mol Biol Cell. 2003;14:5011-8.

\section{Figures}


A

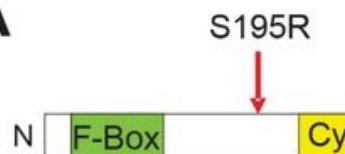

S621G

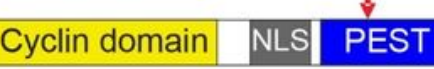

Pseudocatalytic Substrate recruitment module

Regulatory module

C

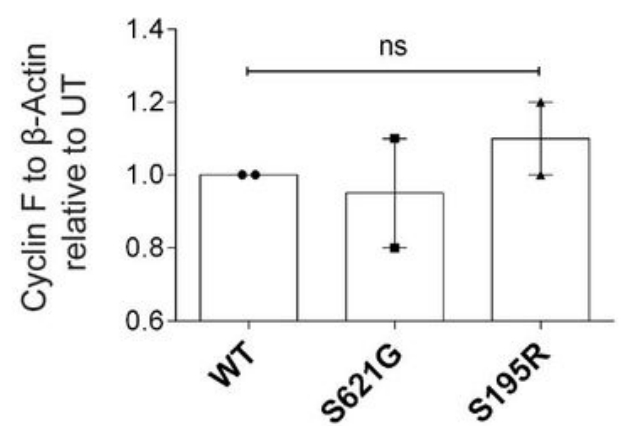

D

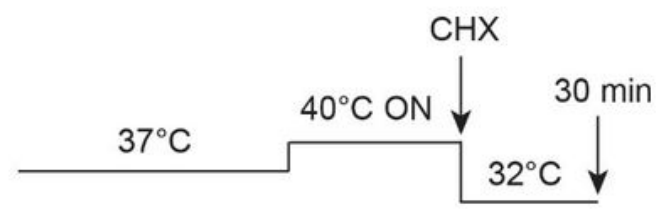

E

$\underline{32^{\circ} \mathrm{C}-\mathrm{ER}}$

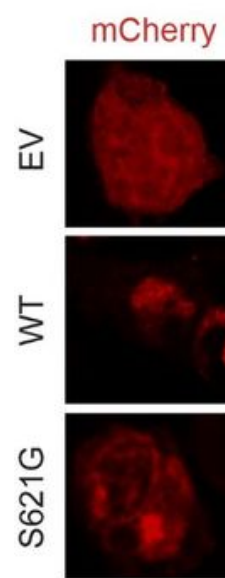

VSVG ${ }^{\mathrm{ts0} 45}$
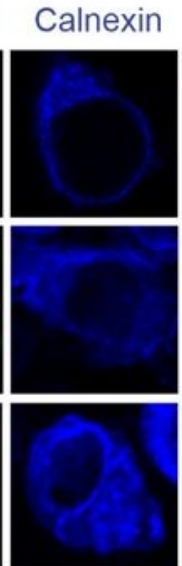

Merge
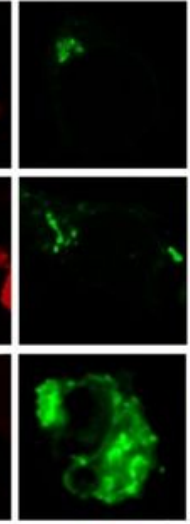

$\mathbf{F}$

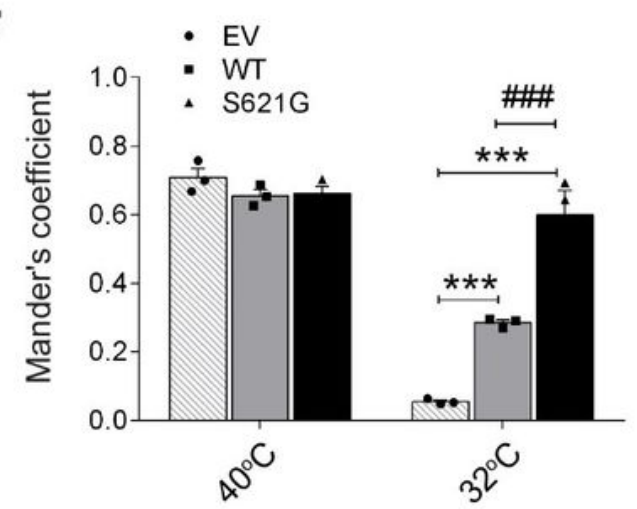

B

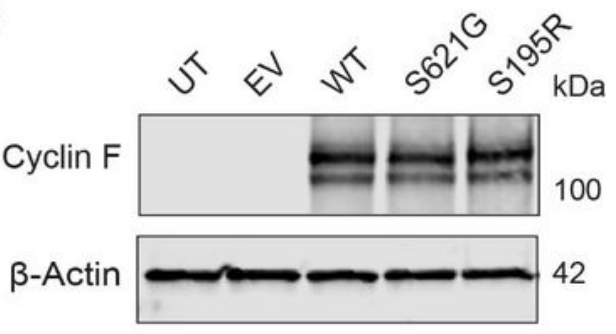

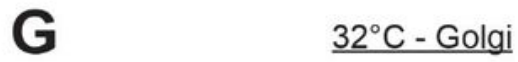

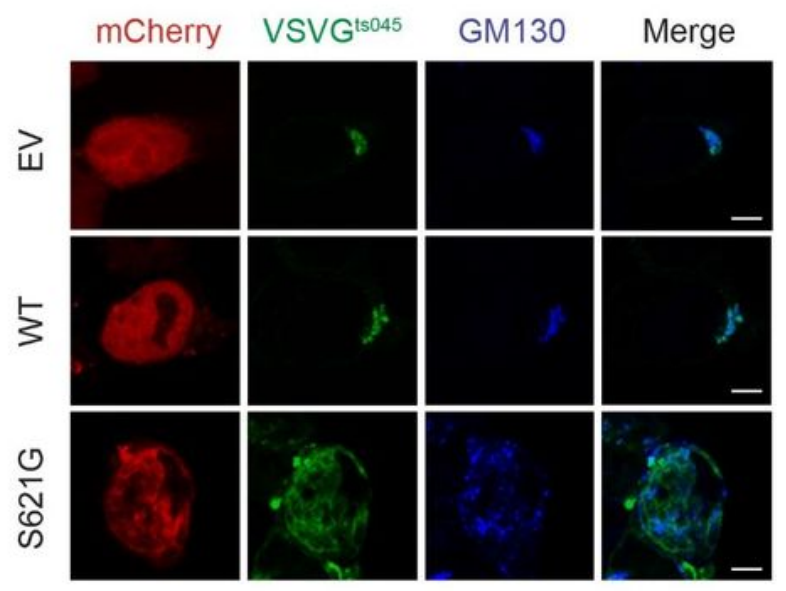

H

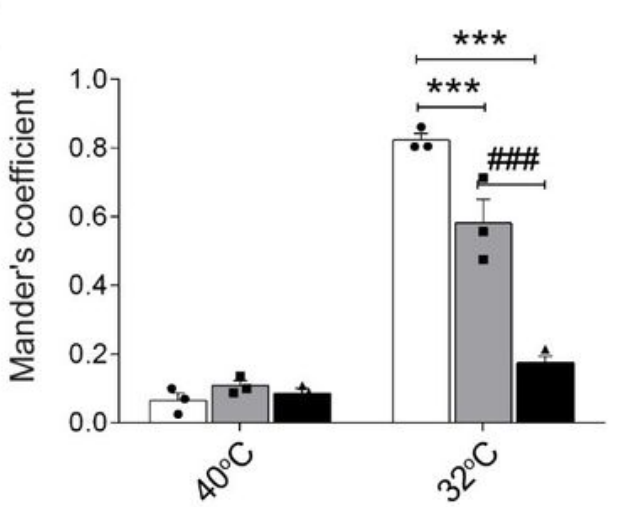

\section{Figure 1}

ALS/FTD mutant cyclin F interferes with ER to Golgi trafficking. (A) Schematic diagram illustrating domain structure of cyclin F showing the locations of the p.S621G mutation in the PEST domain and of the p.S195R mutation between the F-box and the cyclin domain. (B) Western blotting of cyclin $\mathrm{F}$ in lysates prepared from untransfected mouse HEK293 cells (UT) or cells expressing wild-type (WT) or mutant cyclin F in pmCherryC1 vector (EV: empty vector). (C) Relative intensity of cyclin F to $\beta$-Actin in B. Mean \pm 
SEM, symbols represent 2 independent experiments, one-way ANOVA, ns, non-significant. (D) Experimental paradigm: cells expressing VSVGts 045 for $24 \mathrm{~h}$ were incubated at $40^{\circ} \mathrm{C}$ overnight to misfold VSVGts045, leading to its accumulation in the ER. When cells are placed at the permissive temperature $\left(32^{\circ} \mathrm{C}\right)$, VSVGts 045 refolds and transits to the Golgi apparatus within $30 \mathrm{~min}$. (E) Immunocytochemistry of Calnexin in SH-SY5Y cells co-expressing GFP-tagged VSVGts045 (VSVG) and mCherry-tagged empty vector (EV), wild-type (WT) or cyclin FS621G, after incubation at $40^{\circ} \mathrm{C}$ and $32^{\circ} \mathrm{C}$. Scale bar $=5 \mu \mathrm{m}$. $(\mathrm{F}) \mathrm{The}$ degree of co-localisation of VSVGts045 with Calnexin was quantified using Mander's coefficient. (G) Immunocytochemistry of GM130 in undifferentiated SH-SY5Y cells co-expressing GFP-tagged VSVGts 045 and mCherry-tagged EV or WT or cyclin FS621G, after incubation at $40^{\circ} \mathrm{C}$ and $32^{\circ} \mathrm{C}$. Scale bar $=5 \mu \mathrm{m}$. $(\mathrm{H})$ The degree of colocalisation of VSVGts045 with GM130 was quantified using Mander's coefficient. $(F, H)$ Graphs represent mean \pm SEM. Symbols represent 3 independent experiments, two-way ANOVA (factors "temperature" and "genotype") followed by a post-hoc Tukey test for multiple comparisons; ns, non-significant, ${ }^{\star \star \star} p<0.001$ vs cells expressing UT and EV, \#\#\#p<0.001 vs cells expressing cyclin FWT. 
A

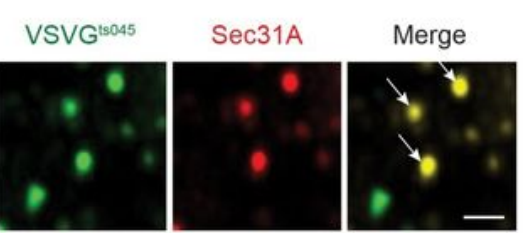

B overnight $40^{\circ} \mathrm{C}$

Cells coexpressing VSVG and cyclin F

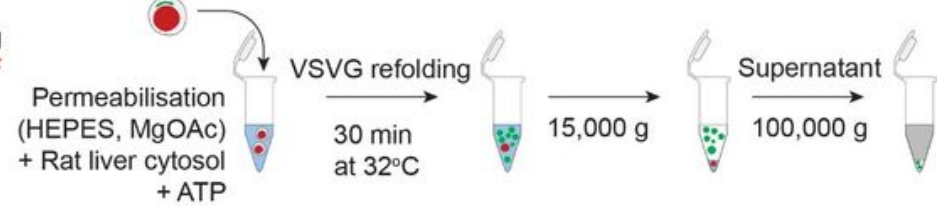

C Before cell-free vesicle budding reaction

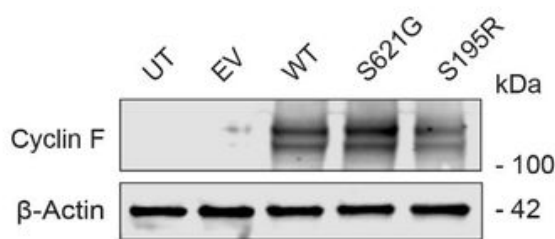

D
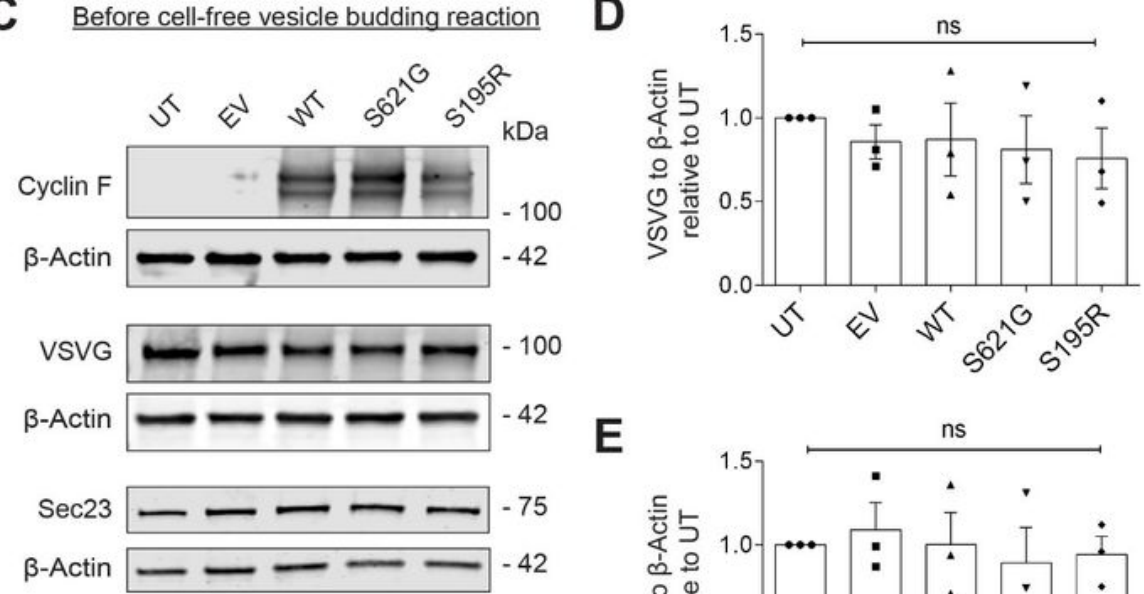

F After cell-free vesicle budding reaction
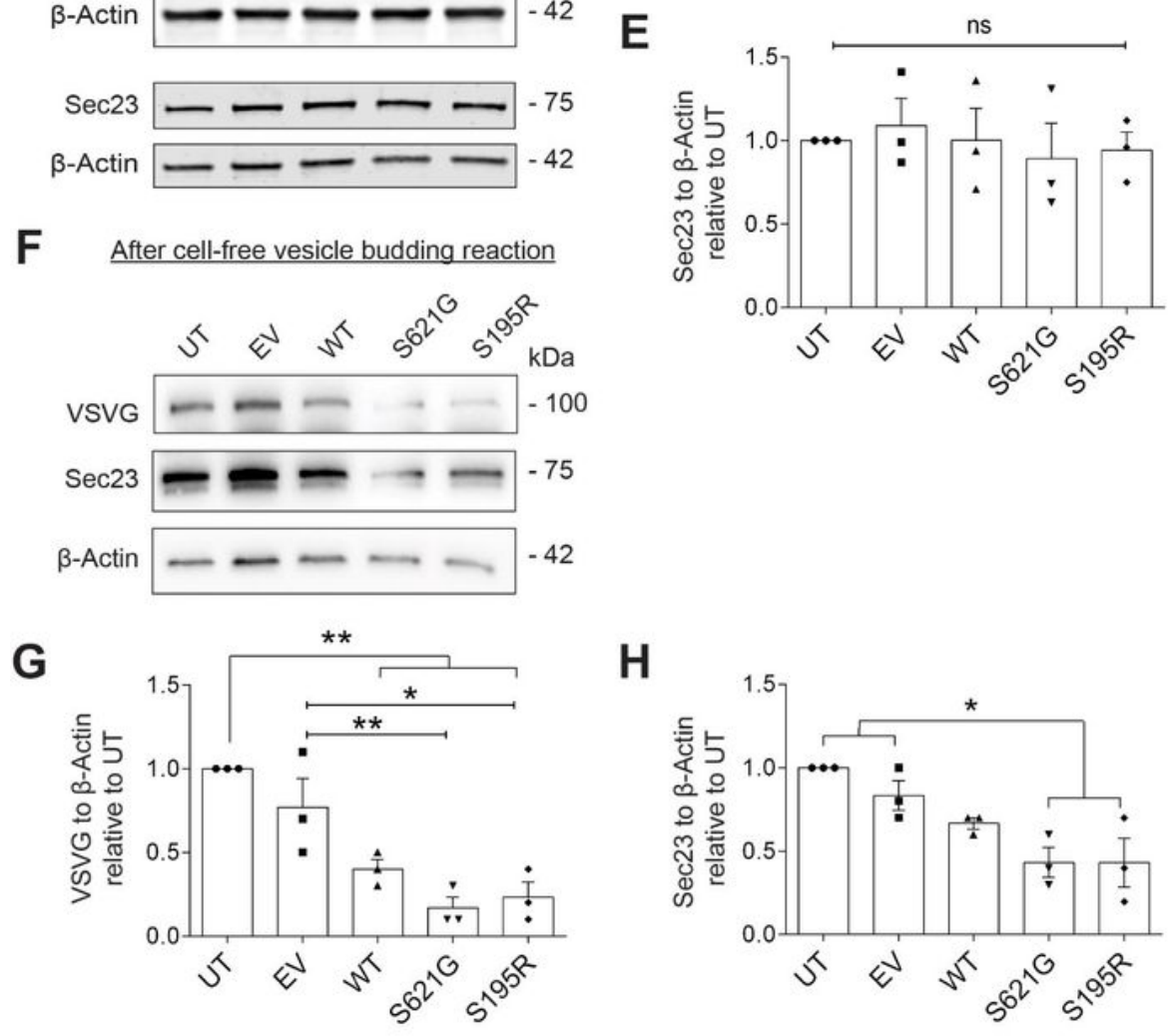

Figure 2

ALS/FTD mutant cyclin F perturbs the budding of COPII vesicles. (A) Immunocytochemistry of Sec31 in SH-SY5Y cells expressing GFP-tagged VSVGts045 (VSVG), after incubation at $40^{\circ} \mathrm{C}$ and $32^{\circ} \mathrm{C}$. Scale bar $=$ $5 \mu \mathrm{m}$. (B) Schematic diagram depicting the experimental procedure used for the cell-free vesicle budding reactions. Briefly, VSVGts045 was trapped in the ER by incubating HEK293T cells overnight at $40^{\circ} \mathrm{C}$ and ER-vesicle budding was reconstituted in vitro in perforated cells expressing $\mathrm{EV}$ or cyclin $\mathrm{F}$ by incubating 
cells at $320 \mathrm{C}$ with rat liver cytosol and an ATP regenerating system. Budded vesicles were collected by centrifugation. (C) Western blotting of cyclin F, VSVGts045 and Sec23 in lysates from UT or cells expressing EV, cyclin FWT or mutant, before ER-derived vesicles were isolated. $\beta$-Actin was used as a loading control for protein expression. (D) Relative intensity of VSVGts045 to $\beta$-Actin (normalized to UT) in C. (E) Relative intensity of Sec23 to $\beta$-Actin (normalized to UT) in $C$ is similar between all groups ( $p>0.05$ ). (F) Budded vesicle preparations were subjected to Western blotting using anti-VSVG and antiSec23 antibodies. $\beta$-Actin was used as a loading control for protein transfer and expression. UT, untransfected cells, EV, empty vector expressing mCherry only. (G) Relative intensity of VSVGts045 in blots in F normalised to $\beta$-Actin and control untransfected cells (UT). (H) Relative intensity of Sec23 in blots in F normalised to $\beta$-Actin and control untransfected cells (UT). (D, E, G, H) Graphs represent mean \pm SEM. Symbols represent 3 independent experiments, one-way ANOVA followed by a post-hoc Tukey test for multiple comparisons, ns, non-significant, ${ }^{*} p<0.05$ and ${ }^{* *} p<0.01$ vs cells expressing UT and EV. 

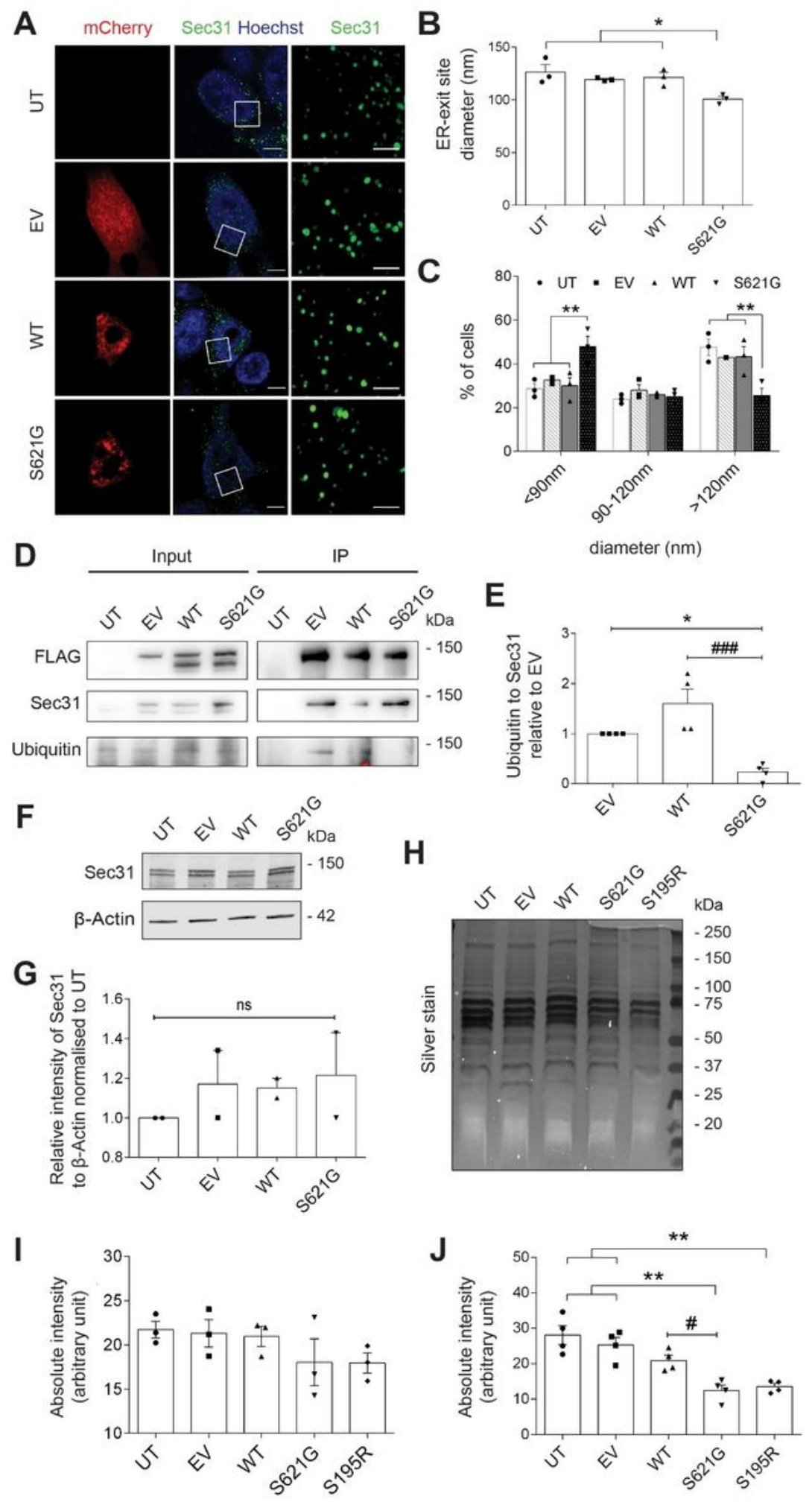

Figure 3

ALS/FTD mutant cyclin F perturbs ER-exit sites and interferes with protein secretion. (A) Fluorescent confocal airyscan microscopy images of HEK293 cells following immunocytochemistry for Sec31 and Hoechst staining in untransfected cells (UT) or cells expressing mCherry only (EV), mCherry-tagged WT or mutant Cyclin FS621G for 48h. Left panel: scale bar $=5 \mu \mathrm{m}$. Right panel: Higher magnification images acquired with Airyscan of the area delimited by white squares on images on left panel. Scale bar $=0.5$ 
$\mu \mathrm{m}$. Each dot represents Sec31-positive ER exit sites. (B) Diameter of ER-exit site clusters calculated from images obtained in A (right panel). For EV, WT and Cyclin FS621G: symbols represent ERES cluster diameter calculated from $n=30$ cells from 3 independent experiments, $* \star * p<0.001$, one-way ANOVA. (C) Proportion of HEK293T cells with ER-exit site diameters below $90 \mathrm{~nm}$; between 90 and $120 \mathrm{~nm}$; and over $120 \mathrm{~nm}$. Mean \pm SEM; symbols represent $\mathrm{n}=3$ independent experiments, ${ }^{*} \mathrm{p}<0.01$ difference vs UT, EV and WT, one-way ANOVA, followed by post-hoc Tukey test. ER exit-site diameter was quantified from 500+ ERES per group from HEK293T cells from 3 independent experiments. The total number of ER-exit sites quantified in each group was UT: $n=1,827 ; E V: n=2,114 ; W T: n=1,722 ; S 621 G: n=1,746$. (D) Immunoprecipitation (I.P.) of FLAG-tagged Sec31 using an anti-FLAG antibody in lysates prepared from cells co-expressing Sec31 and either EV or cyclin F. FLAT-tagged Sec31 was not expressed in untreated samples (UT). Western blotting was performed using anti-FLAG, anti-Sec31 and anti-ubiquitin antibodies. Left panel: input (10 $\mu \mathrm{g}$ of proteins). Right panel: I.P. (E) Relative intensity of ubiquitin to Sec31 of blots in A. (F) Western blotting using an anti-Sec31 antibody in lysates prepared from HEK293T cells expressing empty vector (EV), wild-type (WT) or mutant cyclin FS621G. $\beta$-Actin was used as a loading control for protein expression. (G) Relative intensity of Sec31 to $\beta$-Actin (normalized to UT) in $C$ is similar between all groups $(p>0.05)$. $(H)$ Silver stained SDS-PAGE gel of total proteins secreted into the conditioned medium of untransfected (UT) cells, or cells expressing empty vector (EV) or cyclin $\mathrm{F}$ (72 h post-transfection). (I) Relative intensity of total protein bands in the conditioned medium in E. $(J)$ Relative intensity of protein bands $>100 \mathrm{kDa}$ in the conditioned medium in $\mathrm{E}$. $(\mathrm{B}, \mathrm{C}, \mathrm{E}, \mathrm{G}, \mathrm{I}, \mathrm{J})$ The graph represents mean $\pm \mathrm{SEM}$, oneway ANOVA. Symbols represent $n=2-4$ independent experiments; one-way ANOVA followed by a post-hoc Tukey test for multiple comparisons; ns, non-significant, ${ }^{*} p<0.05$ and ${ }^{* *} p<0.01$ vs UT, EV, $\# p<0.05$ and $\# \#$ \# $<0.001$ vs WT. 


\begin{tabular}{|c|c|c|}
\hline NHK-Venus & Venus & NHK \\
\hline NHK-ddVenus & ddVenus & NHK \\
\hline
\end{tabular}
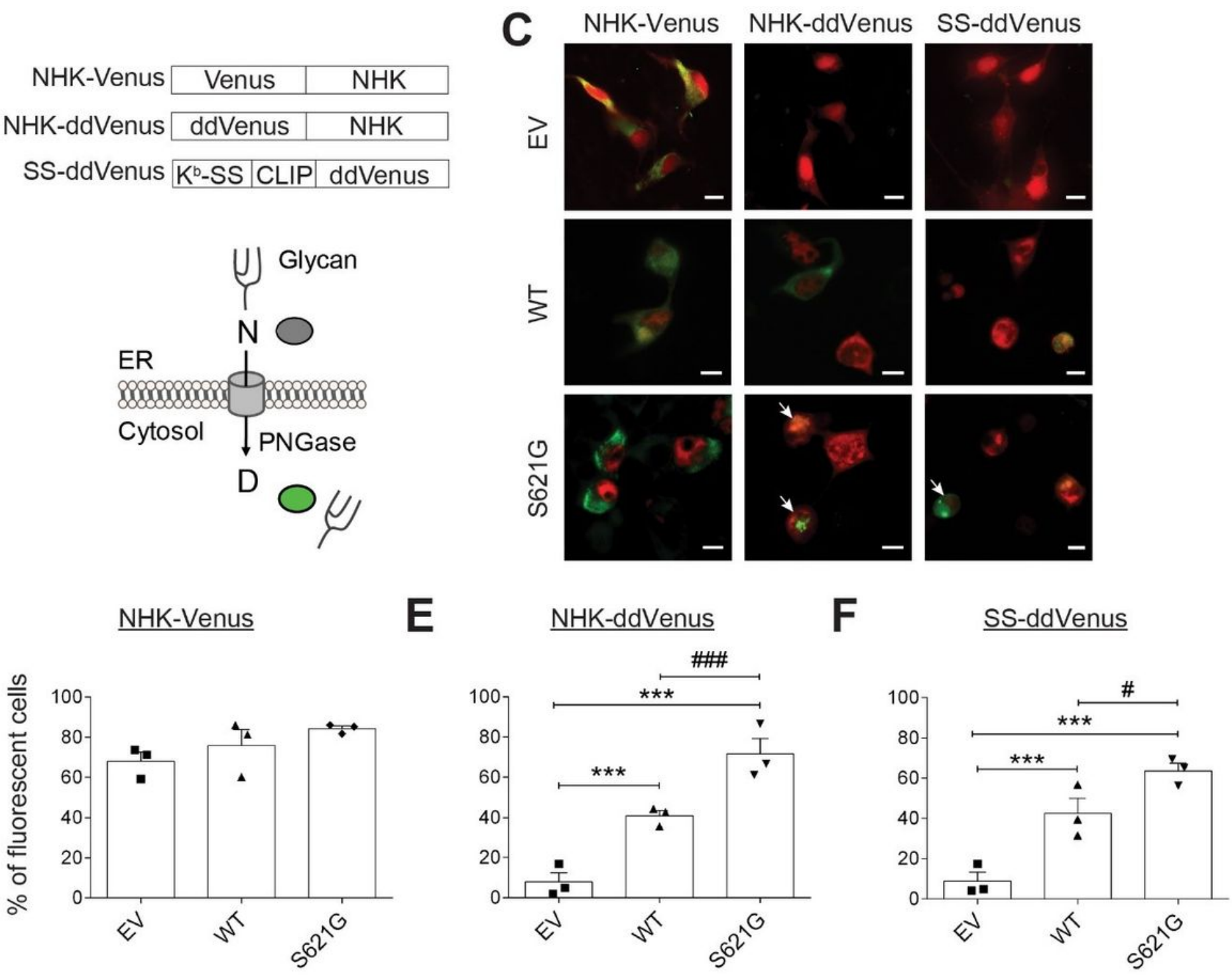

\section{Figure 4}

ALS/FTD mutant cyclin FS621G impedes ERAD. (A) Fluorescent ERAD substrates used to monitor ERAD. Wild-type Venus and mutant ddVenus C-terminal, in which Asn was substituted for Asp at position 82 (SS-ddVenus), were fused to a well-known ERAD substrate, the unstable null Hong Kong variant of alpha1-antitrypsin (NHK-Venus; NHK-ddVenus)32,33. The SS-ddVenus construct contains an ER-targeted signal sequence (Kb-SS) fused to dd-Venus. (B) The system is based on glycosylation and deglycosylation of Venus protein. Removal of a glycan by the cytosolic enzyme Peptide:N'glycanase (PNGase) results in deamidation of the glycosylated Asn, converting it to an Asp residue. The fluorescence results from protein that both reaches the ER and also retrotranslocates to the cytosol. Both glycosylation and deglycosylation (ER entry and exit) are required for fluorescence33. (C) Representative fluorescent microscopy images of SH-SY5Y cells expressing empty vector (EV) and cyclin F with ERAD substrates (arrows). NHK fused to Venus was used as a control for cell transfection. Scale bar $=10 \mu \mathrm{m}$. (D-F) The proportion of cells co-expressing CCNF with NHK-Venus (D), NHK-ddVenus (E) or SS-ddVenus (F) relative to the total number of mCherry-fluorescent cells was quantified. Graphs represent median 
mean \pm SEM. Symbols represent three independent experiments, ${ }^{* \star *} p<0.001$ difference vs EV; \#p<0.05, \#\#\#p<0.001 difference vs WT, one-way ANOVA followed by a post-hoc Tukey test for multiple comparisons.

A

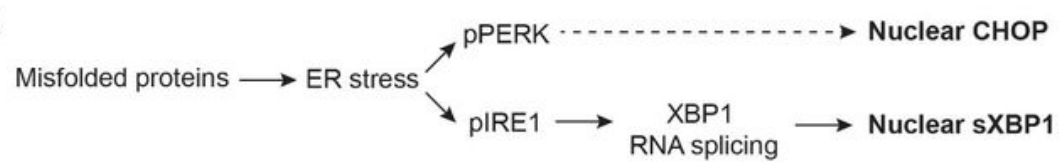

B

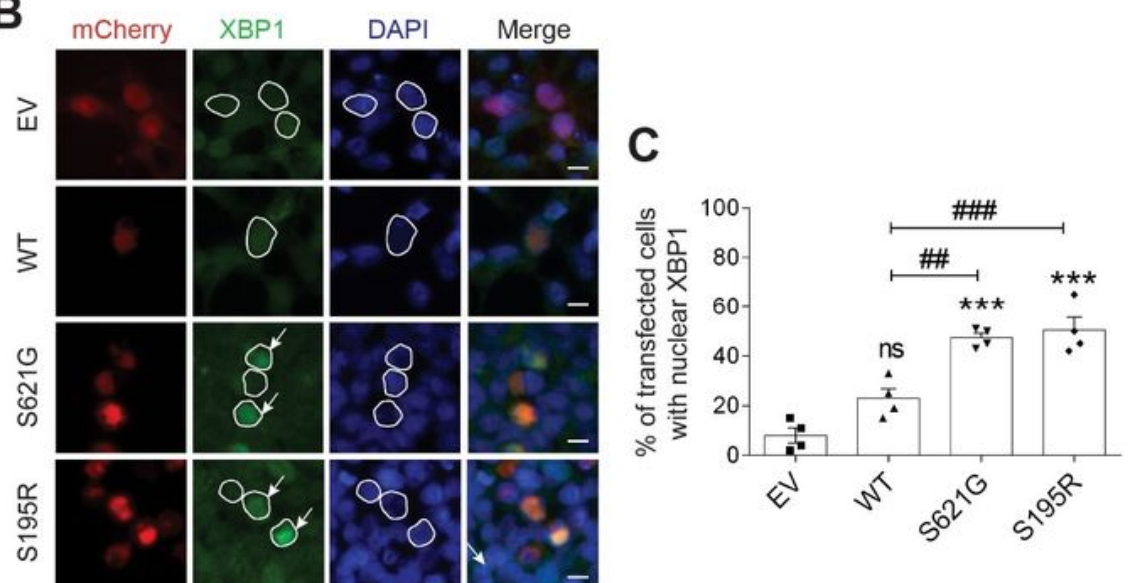

D mCherry

CHOP

DAPI Merge
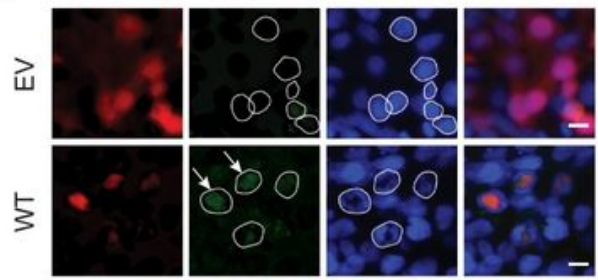

E
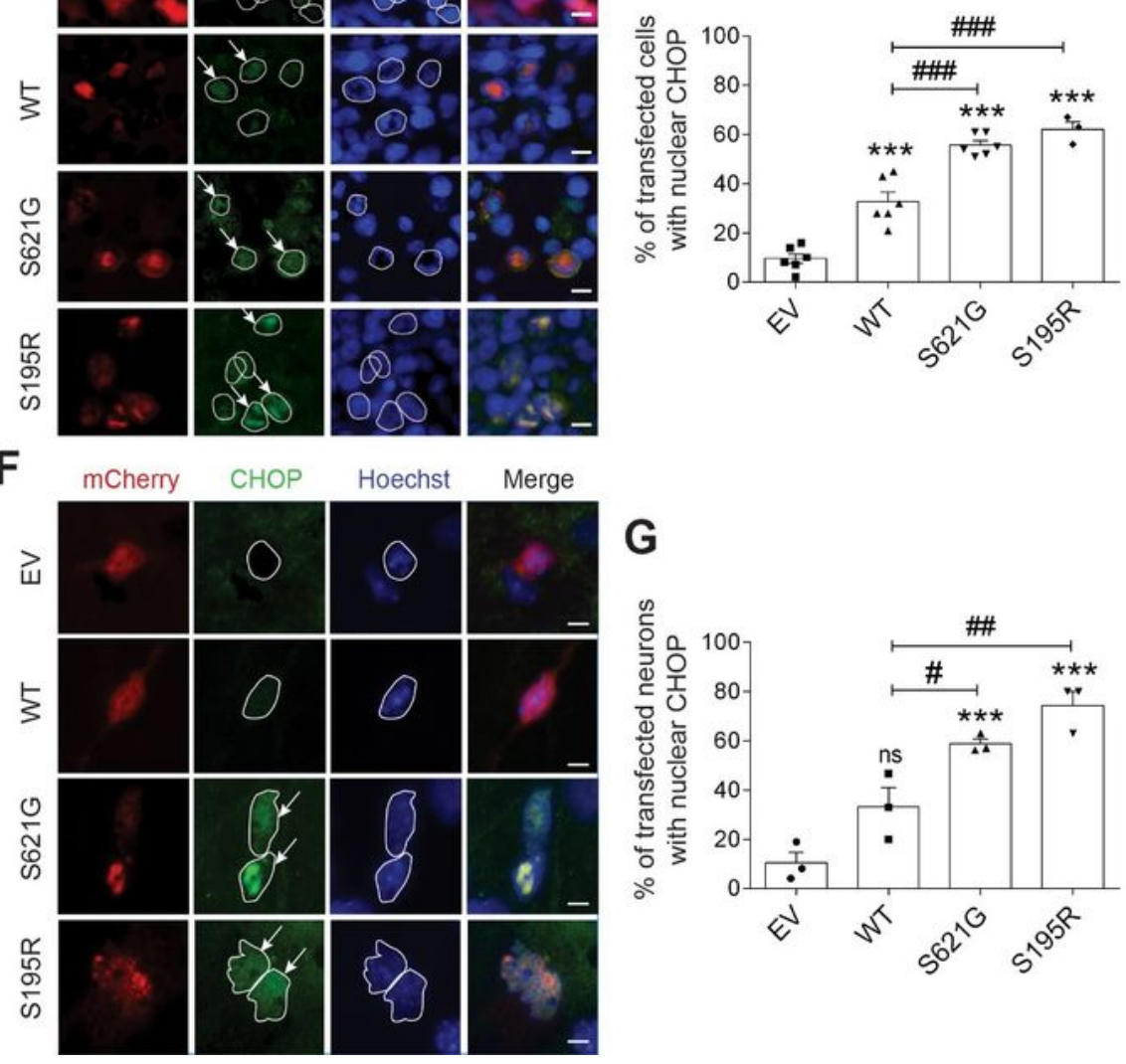

Figure 5

ALS/FTD mutant cyclin F induces ER stress (A) Schematic diagram representing the UPR pathway. Misfolded proteins in the ER accumulate, induce ER stress and activate the UPR. UPR modulators PERK 
and IRE1 are activated by dimerization followed by phosphorylation, inducing activation of the UPR transcription factor XBP1, resulting in production of its spliced form (sXBP1). Under long-term ER stress, the UPR pro-apoptotic pathway is triggered, leading to the activation of the transcription factor $\mathrm{CHOP}$ and expression of apoptotic UPR genes45. PERK, protein kinase R-like endoplasmic reticulum kinase; $\mathrm{CHOP}$, C/EBP-homologous protein; IRE1a, inositol-requiring protein 1a; XBP1, X-box binding protein 1. (B) Representative fluorescence microscopy images of HEK293 cells expressing mCherry-tagged cyclin F, following immunocytochemistry for XBP1s and Hoechst staining in cells expressing EV, WT or mutant cyclin F. Arrows: cells with nuclear XBP1 immunoreactivity. Scale bar $=10 \mu \mathrm{m}$. (C) Proportion of cells with nuclear XBP1s immunoreactivity was quantified. (D) Representative fluorescence microscopy images of HEK293T cells expressing mCherry-tagged cyclin F, following immunocytochemistry for CHOP and Hoechst staining in cells expressing EV, WT or mutant cyclin F. Arrows: cells with nuclear CHOP immunoreactivity. Scale bar $=10 \mu \mathrm{m}$. (E) Proportion of cells with nuclear CHOP immunoreactivity was quantified. (F) Representative fluorescence microscopy images of mouse primary cortical neurons expressing mCherry-tagged cyclin $\mathrm{F}$, following immunocytochemistry for $\mathrm{CHOP}$ and Hoechst staining in neurons expressing EV, WT or mutant cyclin F. Arrows: neurons with nuclear CHOP immunoreactivity. Scale bar $=10 \mu \mathrm{m}$. (G) The proportion of neurons with nuclear CHOP immunoreactivity was quantified. $(C, E, G)$ Graphs represent mean \pm SEM. Symbols represent 3-6 independent experiments, $(C, E) n=50+$ cells per group, $(G) n=10-30$ neurons per group; one-way ANOVA followed by a post-hoc Tukey test for multiple comparisons; ns, non-significant, ${ }^{\star \star *} p<0.001$ vs UT and $E V ; \# p<0.05$, \#\#p<0.01 and \#\#\#p<0.001 vs cells expressing cyclin FWT. Nuclei are defined with a white outline. 


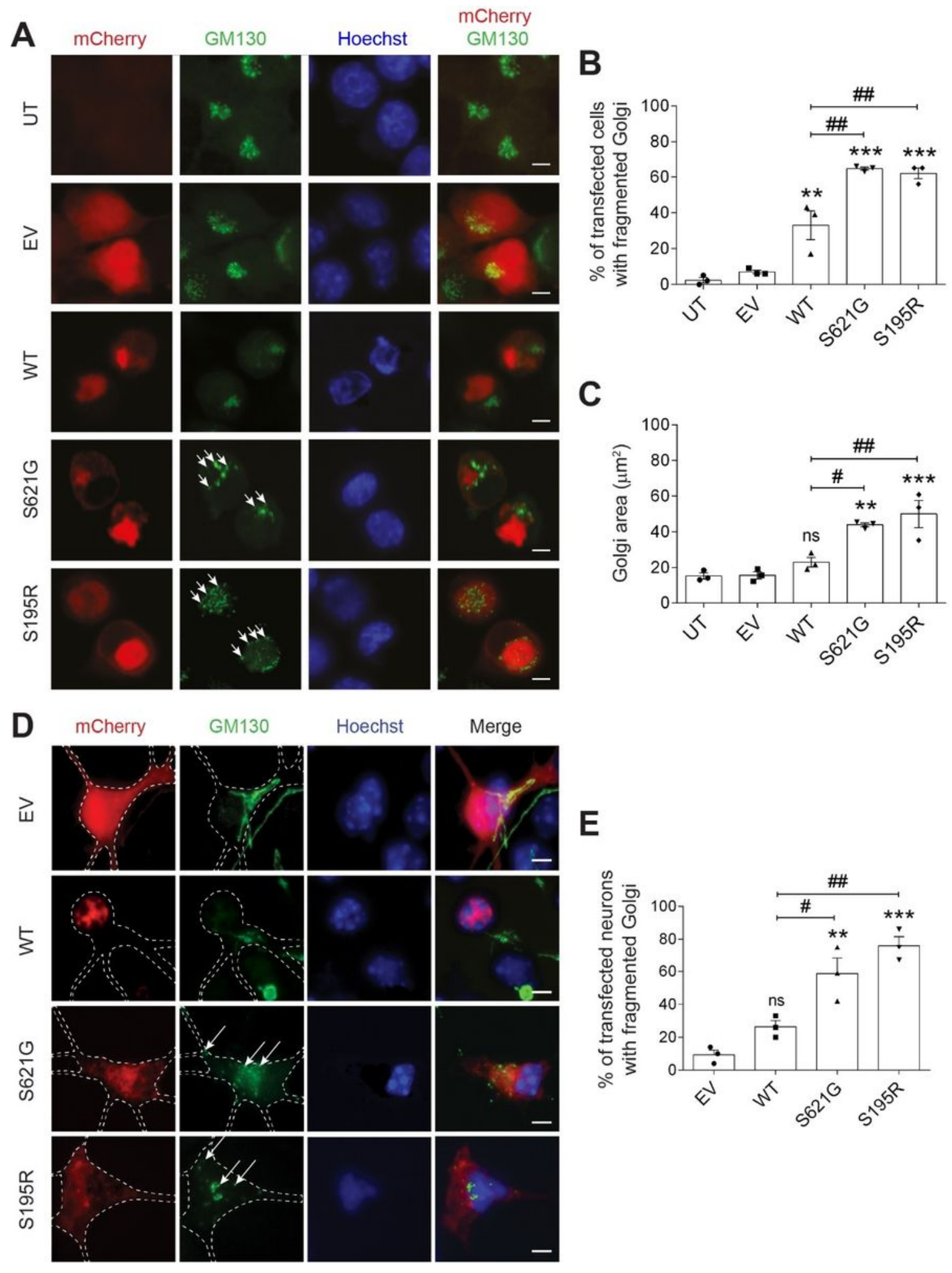

Figure 6

ALS/FTD mutant cyclin F induces Golgi fragmentation (A) Fluorescent confocal microscopy images of EV or cyclin F, following immunocytochemistry for GM130 and Hoechst staining, of SH-SY5Y cells expressing EV or cyclin F. Arrows: Golgi fragments. Scale bar $=10 \mu \mathrm{m}(\mathrm{B})$ The proportion of cells with fragmented Golgi in A was quantified. (C) The area covered by Golgi fragments was measured in each cell. (D) Fluorescent confocal microscopy images of empty vector (EV) or cyclin F, GM130 and Hoechst in 
mouse primary cortical neurons expressing EV or cyclin F. Arrows: Golgi fragments. The dashed white line delimits the outline of the neuron. Scale bar $=5 \mu \mathrm{m}$. (E) The proportion of primary neurons with fragmented Golgi was quantified. (B, C, E) Graphs represent mean \pm SEM. Symbols represent 3 independent experiments, $(B, C) n=50+$ cells per group from, $(E) n=10-30$ neurons per group; one-way ANOVA followed by a post-hoc Tukey test for multiple comparisons; ns, non-significant, ${ }^{* *} \mathrm{p}<0.01$ and $* * \star p<0.001$ vs UT and $E V ; \# p<0.05$ and $\# \# p<0.01$ vs cells expressing cyclin FWT.
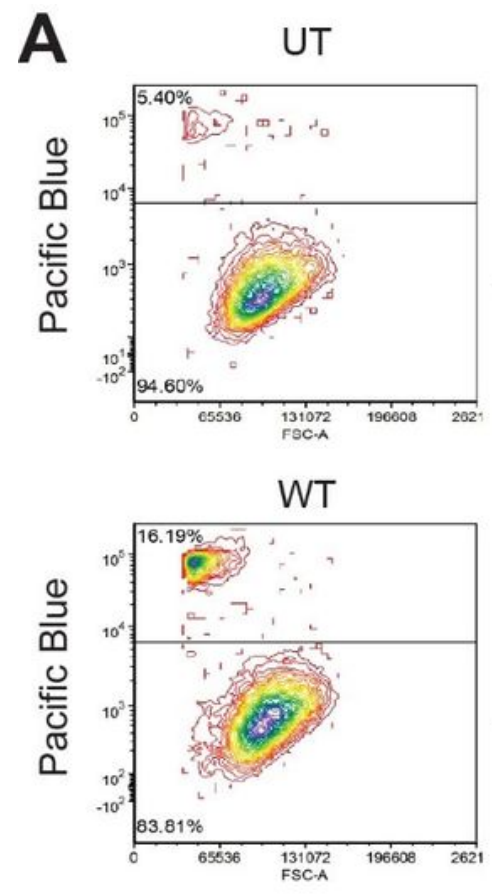

EV

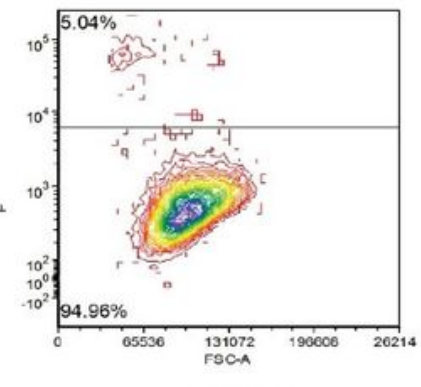

S621G

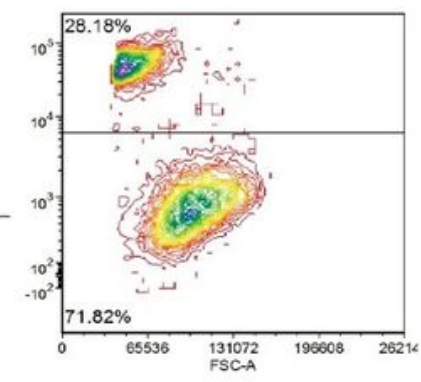

Merge

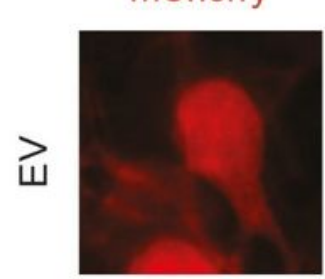

Hoechst
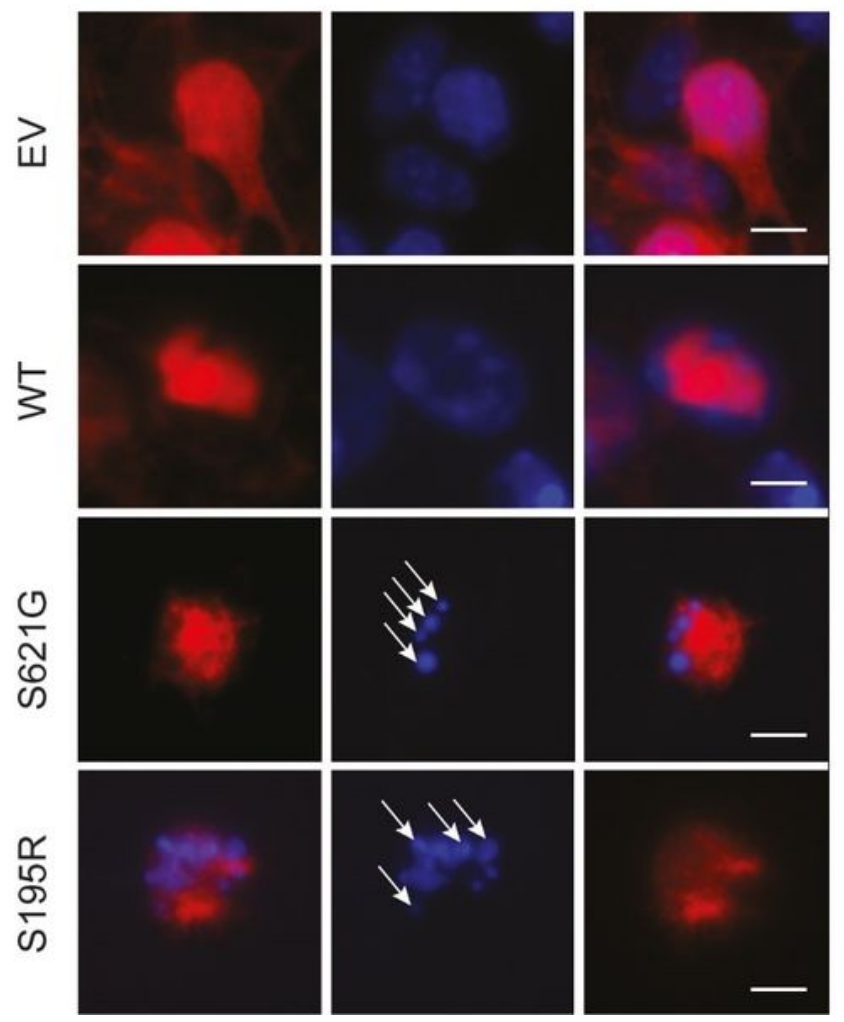

B

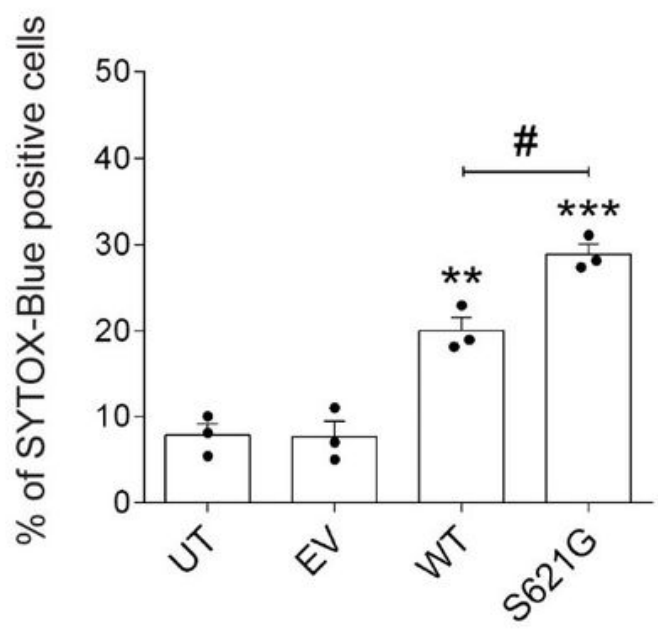

D

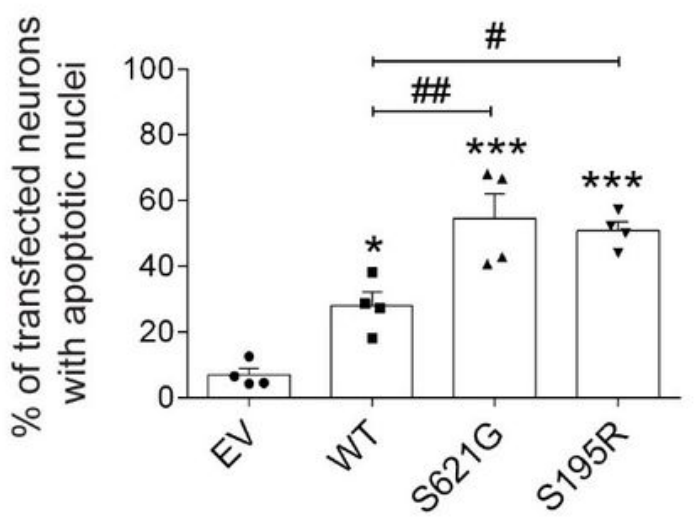

Figure 7 
ALS/FTD mutant cyclin F induces cellular death (A) Flow cytometry analysis of SH-SY5Y cells expressing mCherry-tagged cyclin F after staining with Sytox Blue, a marker for dead cells. UT, untransfected, EV, empty vector, WT, wild-type. (B) Quantification of Sytox Blue positive cells. (C) Representative fluorescent microscopy images of mouse cortical primary neurons expressing cyclin F, stained with Hoechst.

Condensed nuclei (arrows), indicative of neuronal death, are present in cells expressing mutant cyclin $\mathrm{F}$. Scale bar $=10 \mu \mathrm{m}$. (D) The proportion of cells with condensed nucleus fragments was quantified. $(B, D)$ Graphs represent mean \pm SEM. Circles represent $n=3-4$ independent experiments, one-way ANOVA, followed by a post-hoc Tukey test for multiple comparisons, ${ }^{*} p<0.05$, ${ }^{* *} p<0.001,{ }^{* \star \star} p<0.001$ compared to UT and $E V ; \# p<0.05$ and \#\#p<0.01 compared to cyclin FWT.

\section{NORMAL}

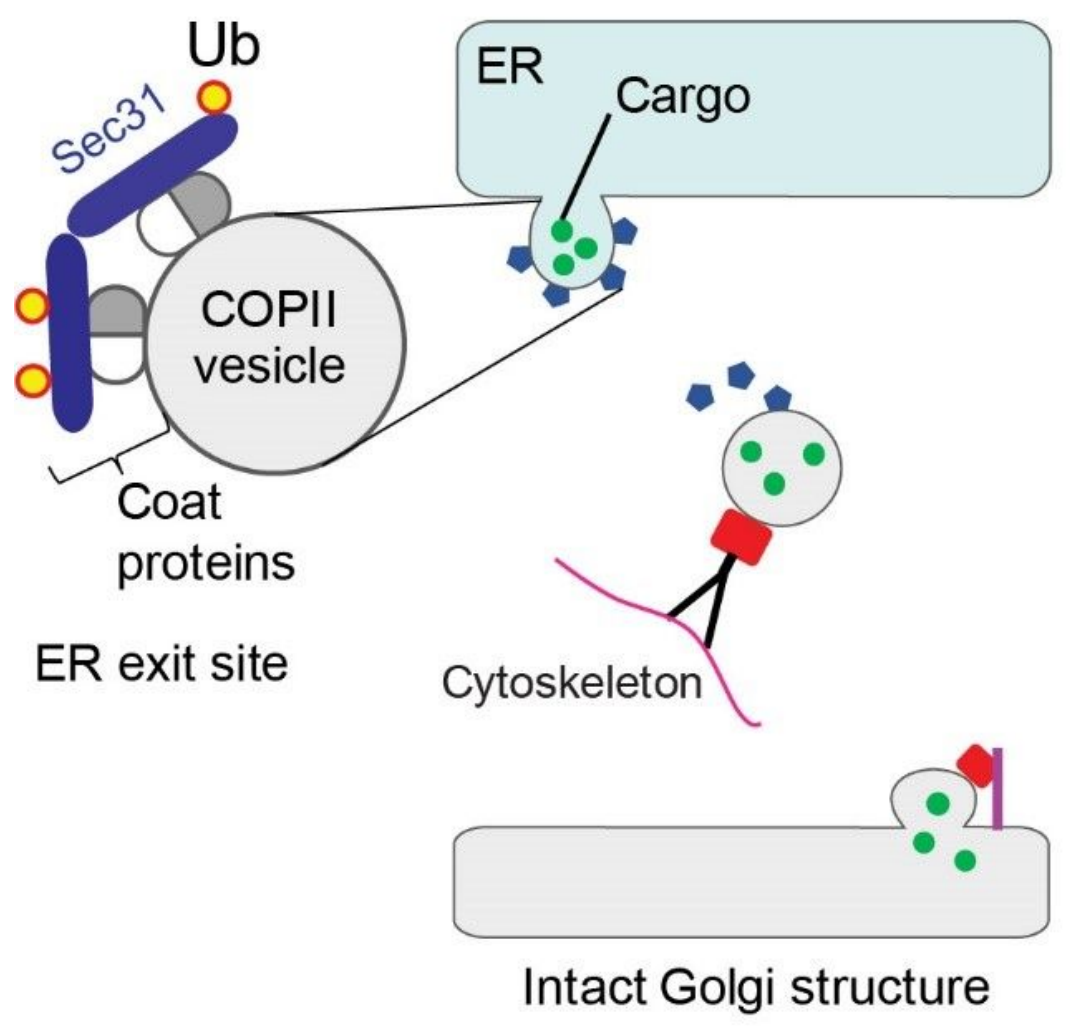

ALS
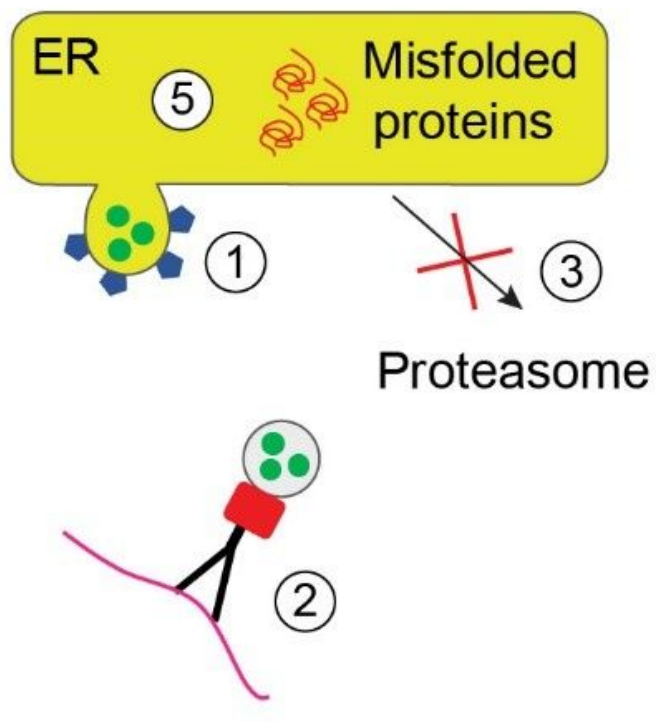

(4)

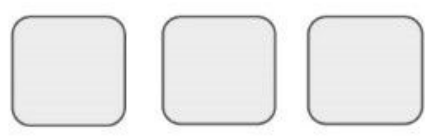

Fragmented Golgi
Pathological events in ALS

(1) Atypical ER exit sites

(2) ER to Golgi transport defect

(3) ERAD dysfunction

(4) Golgi fragmentation

(5) ER stress 
Schematic diagram based on the findings of this study, illustrating a possible mechanism to explain of how mutant cyclin $\mathrm{F}$ impairs ER homeostasis in neuronal cells, leading to apoptotic cell death in ALS. Misfolded cyclin F triggers the formation of abnormal ER-derived vesicles (1), which inhibits ER-Golgi transport (2), ERAD and proteasomal function (3), triggering Golgi fragmentation (4), ER stress (5) and apoptotic cell death.

\section{Supplementary Files}

This is a list of supplementary files associated with this preprint. Click to download.

- SupplementaryFigure.docx 